\title{
Aeroelastic Stability Analysis of a Full-Scale Isolated Proprotor on the Tiltrotor Test Rig
}

\author{
Sesi Kottapalli, Carl R. Russell, C. W. Acree, Jr., and Thomas R. Norman \\ Aeromechanics Office \\ NASA Ames Research Center, Moffett Field, CA, 94035, USA
}

\begin{abstract}
A full-scale isolated proprotor test was recently conducted in the USAF National Full-Scale Aerodynamics Complex (NFAC) 40- by 80-Foot Wind Tunnel at NASA Ames. The test article was a 3-bladed research rotor derived from the right-hand rotor of the AW609. For this test, the NASA Tiltrotor Test Rig (TTR) and rotor were installed in the 40- by 80-Foot Wind Tunnel. This paper covers the analyses and testing done to prepare for a safe entry. Included are brief descriptions of the following: NASTRAN models of the TTR, ground vibration tests of the TTR (and resulting modal data), loads analyses, and stability predictions using the comprehensive analysis CAMRAD II. The evolution of these analyses from early in the TTR program until the initiation of actual testing is also discussed. The intent is to show how all of these efforts were integrated to ensure a successful test. This paper includes stability predictions based on NASTRAN modal data and worst-case damping test data. The stability predictions covered all test conditions: hover, cruise (airplane mode), conversion, and helicopter mode. The predictions showed that the TTR and rotor are stable within the test envelope.
\end{abstract}

\section{Nomenclature}

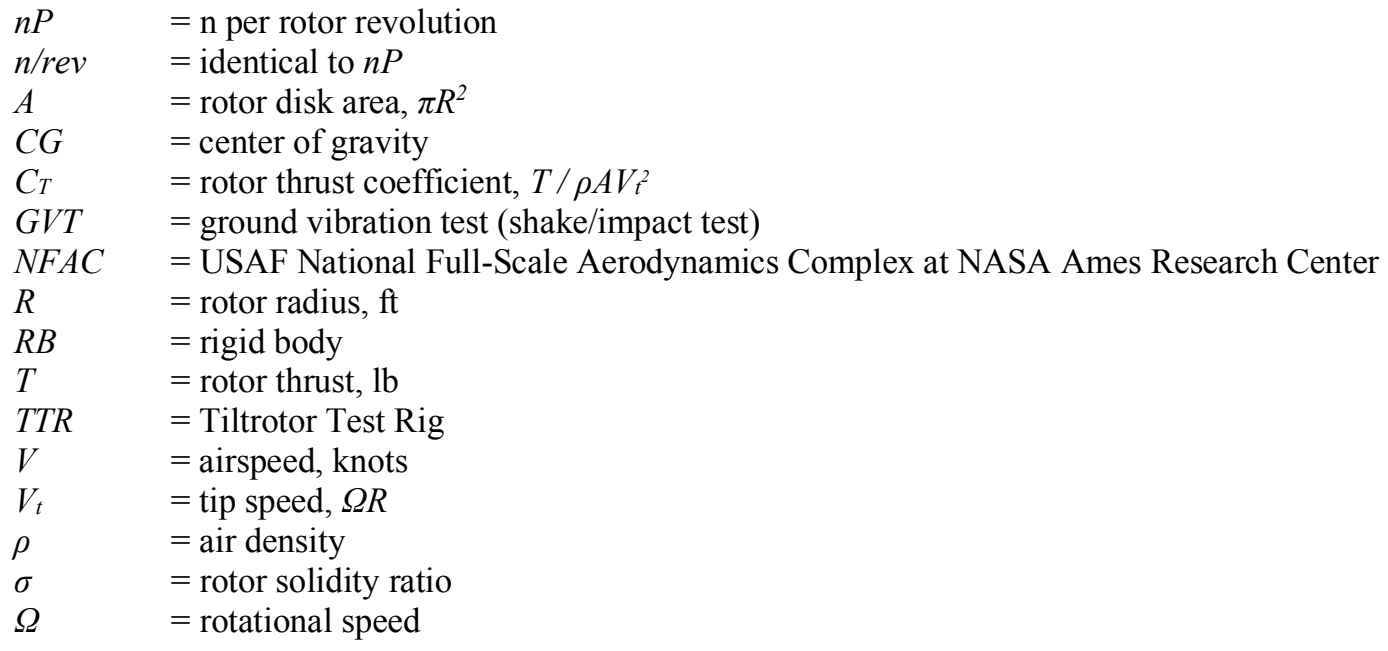

\section{Introduction}

A full-scale isolated proprotor test was recently conducted in the USAF National Full-Scale Aerodynamics Complex (NFAC) 40- by 80-Foot Wind Tunnel at NASA Ames. The test article was a 3-bladed research rotor; in this study, this research rotor is referred to as "699". For this test, the NASA Tiltrotor Test Rig (TTR) and the 699 were installed in the 40x80 wind tunnel. This paper covers the analyses and testing done to prepare for a safe TTR/699 entry. Stability analyses are emphasized, but performance and loads analyses are also included because they define the operating limits within which stability must be assured.

A substantial portion of the research reported in this paper represents follow-on work to two earlier 2018 studies on the TTR/699: a paper on TTR modal test and analysis, Ref. [1], and a companion, analytical paper on TTR/699 whirl flutter stability prediction, Ref. [2]. As is the usual procedure for rotor test stand analysis, modal data from Ref. [1] were used for the stability predictions reported in Ref. [2]. A complete description of the TTR project can be obtained from the following references, some of which are yet to be presented: Ref. [3] will present an overview of 
the entire TTR/699 test program; Ref. [4], an earlier paper, addresses the development and initial testing of the TTR; and Ref. [5] will present correlation results for the full-scale isolated 699 proprotor performance and loads (using newly acquired test data, described below). Background information on the TTR effort at NASA Ames can be found at Ref. [6].

The following is a brief description of the checkout test of the TTR with the 699 proprotor installed on the TTR. The 699 is a 3-bladed research rotor derived from the right-hand rotor of the AW609; this rotor was manufactured by Bell Helicopter under contract to NASA. The 699 was referred to as ' 609 ' in earlier publications, notably Refs. [1] and [2]. The initial checkout test is an integral part of the development of the TTR, whose purpose is to test advanced, full-scale proprotors in the NFAC (Refs. [3-4]). The primary purpose of the just completed checkout test was to demonstrate the operational capability of the TTR over a wide range of test conditions. A secondary goal was to safely collect as much research data as possible. Figure 1 shows the TTR/699 installed in the NFAC 40- by 80-Foot wind tunnel test section. The TTR rotor axis is horizontal and the rig rotates in yaw on the wind tunnel turntable for conversion between airplane and helicopter mode testing. During wind tunnel checkout testing in August 2018, the TTR reached a maximum cruise (airplane mode, 0 -deg yaw) airspeed of 273 knots which is the current NFAC limit. This is the highest airspeed ever achieved by a full-scale proprotor in any wind tunnel.

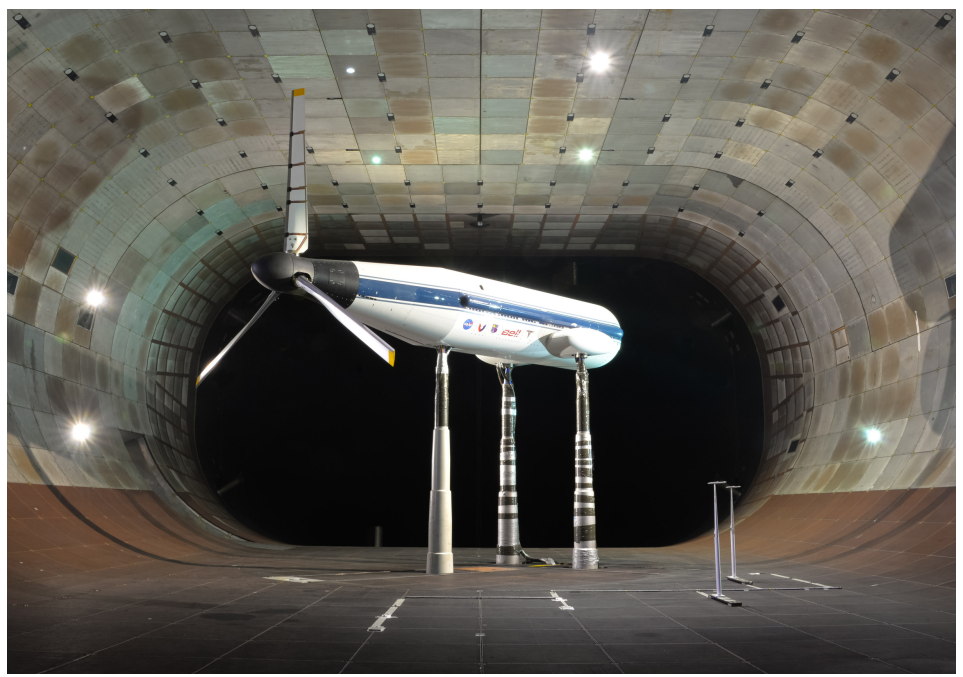

Fig. 1 TTR/699 installed in the USAF NFAC 40- by 80-Foot wind tunnel test section.

The general TTR research areas in proprotor aeromechanics include:

- Performance and efficiency in hover, transition and forward flight

- Vibration and dynamic loads for all flight regimes

- Hub and control loads for all flight regimes

- Variable-frequency dynamics (wide RPM range)

The supporting research opportunities are acoustics measurements and tunnel blockage effects.

A wind tunnel test can safely collect data for operating conditions beyond an aircraft flight envelope. The TTR has a large excess of available power. With all of its four motors operating, the present TTR operational power range extends up to $5000 \mathrm{hp}$, which is far greater than that of the AW609 aircraft. Also, in flight, cruise thrust is much lower than in hover, so the wind tunnel thrust limits do not necessarily apply.

Covered in this paper are the following descriptions and the analytical and experimental studies that were performed to prepare for a safe TTR entry:

- 699 research rotor and TTR

- CAMRAD II model

- TTR NASTRAN models

- Experimental efforts (GVT - ground vibration test: shake and impact tests) 
- Stability predictions (based on NASTRAN and experimental modal data)

The GVT included random and swept-sine waveform excitation (shake tests) and impact excitation.

Photogrammetry techniques were used to measure the effective stiffness and linearity of the wind tunnel strut deflections under load and were used to update and improve the NASTRAN predictions; this is discussed further in the section on NASTRAN models (Ref. [10] has more information on the application of the photogrammetry technique to the TTR rotor balance calibration).

It should be noted that the experimental modal data-based stability predictions performed by NASA (reported in full here and in part previously in Ref. [2]) were preceded by stability predictions performed by Bell. Bell had originally predicted TTR/699 stability in cruise (airplane mode, 0-deg TTR yaw) using the then available NASTRAN modes of the TTR and their ASAP program; those predictions were based on an older design of the test section strut carriage. Furthermore, the Bell predictions were made before new strut tips were designed and fabricated for the TTR. A new strut carriage was expected to have a significant effect on stability, which was the primary motivation for updating the Bell analyses. Establishment of an in-house comprehensive model was another important motivation. More details are given later in the section on "NASTRAN models of the TTR." As noted above, the Bell analyses used ASAP, whereas the present NASA analyses use CAMRAD II (Refs. [7-9]). Furthermore, the current stability analyses address the entire wind tunnel test envelope: cruise (airplane mode, 0-deg yaw), conversion mode (transition, 45-deg yaw specifically), and the helicopter mode (90-deg yaw).

\section{699 Research Rotor and Tiltrotor Test Rig}

Brief descriptions of the 699 research proprotor and the TTR are given below.

\section{A. 699 research rotor}

The 3-bladed rotor has non-linear twist and square tips, the rotor diameter is $26 \mathrm{ft}$ and the geometric solidity is 0.097. The rotor is stiff in-plane with a gimballed hub and yoke (flexbeam). The conversion and helicopter mode rpm is $569(100 \%)$ and the airplane mode (cruise) rpm is $478(84 \%)$. The highest $3 \mathrm{P}(3 / \mathrm{rev})$ frequency is then $28.45 \mathrm{~Hz}$, hence the structural modes of interest range from 0 to $40 \mathrm{~Hz}$.

The 699 rotor is based on the AW609 rotor, and was manufactured by Bell Helicopter under contract to NASA. The main differences between the research and flight rotors are listed below. The research rotor:

- Does not have deicing capability

- Has additional instrumentation

- Has a different pitch horn arrangement, specific to the TTR control system

- Does not have pendulum absorbers

\section{B. Tiltrotor Test Rig (TTR)}

The TTR is a NASA project, joint with the U.S. Army and Air Force, to develop a new, large-scale proprotor test system for the NFAC. The TTR is designed for use in both the 40 - by 80 - and 80 - by 120 -foot NFAC wind tunnels. The rotor axis is horizontal and the rig rotates in yaw on the wind tunnel turntable. The TTR is capable of testing various rotor types: articulated, gimballed, soft in-plane and hingeless with rotor diameters up to $26 \mathrm{ft}$, the 40x80 (40by 80 -Foot) test section limit for good data.

For illustrative purposes, Fig. 2 shows the TTR installed in the 40x80 test section, as modeled by NASTRAN. The complexity of the structure is evident. The three struts and the T-frame can also be seen. The TTR is supported by the struts, which rest on the T-frame; the T-frame is below the floor of the tunnel, which is on the turntable. Figure 2 also shows the mode shape for the first TTR mode (nose yaw). The nose yaw mode and the TTR NASTRAN model are discussed in more detail in a later section.

More information on the TTR test objectives in general can be found in "TTR Overview Presentation" (see Ref. [6]), and is outlined as follows:

- Fill gaps in existing tilt-rotor experimental databases by testing for classic rotor performance, including:

- Hover, up to and beyond stall

- Axial flow beyond 200 knots (up to the 300-knot NFAC limit)

$\circ$ Helicopter and transition modes up to 150 knots (includes edgewise flight) 
- Conduct extended testing beyond the aircraft flight envelope, such as:

○ Slowed rotor in cruise for efficiency (50-75\% hover tip speed)

- Slowed rotor in hover for low noise ( $85 \%$ tip speed)

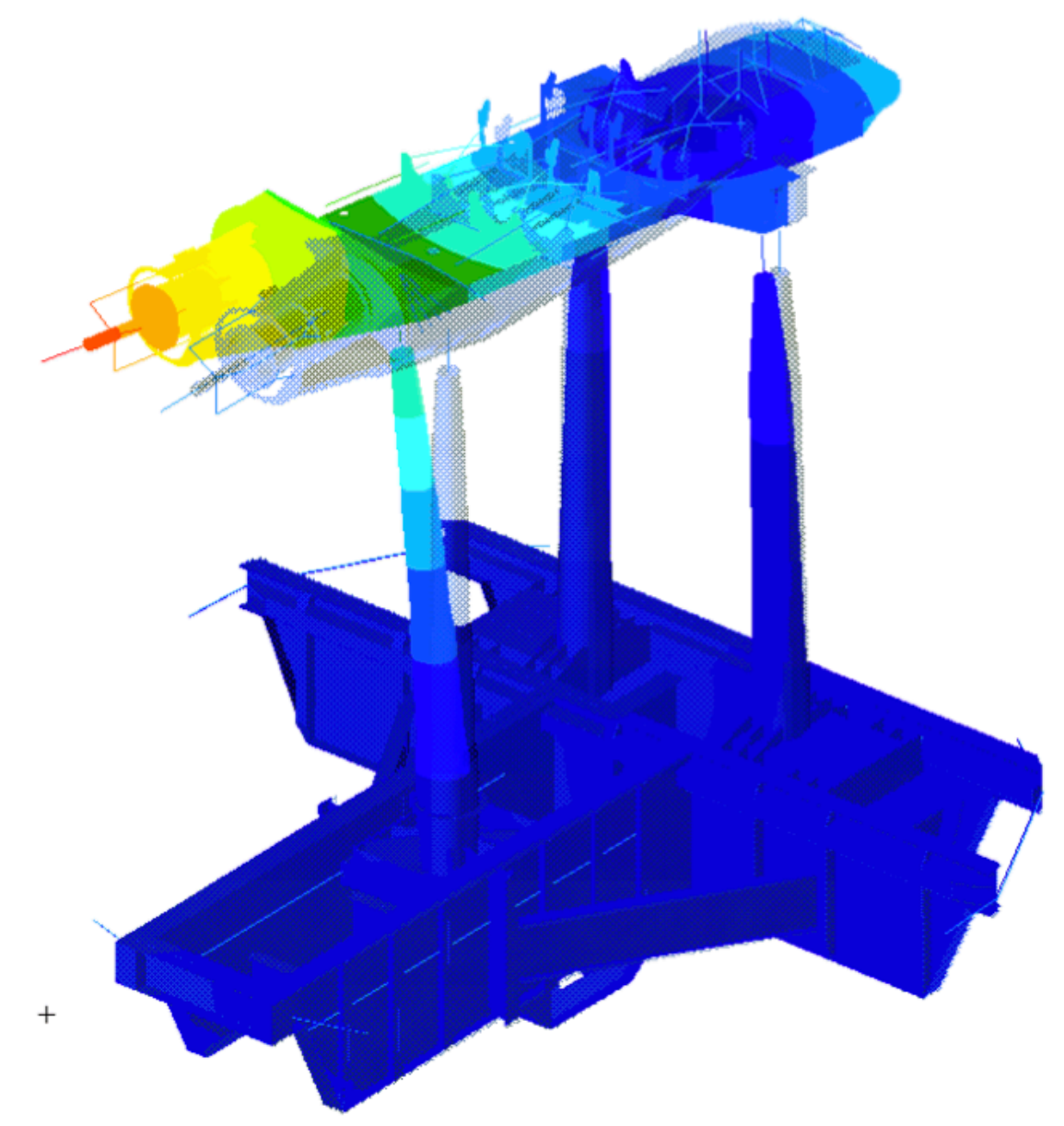

Fig. 2 NASTRAN model of the TTR in the 40x80 wind tunnel (first mode shape - nose yaw).

\section{Analytical Model}

The rotorcraft comprehensive analysis CAMRAD II Release 4.9, Refs. 7-9, was used for the analytical predictions. Table 1 from Ref. [2] summarizes the analytical models used for the performance and loads task (Ref. [2], Ref. [5]) and the currently relevant stability task. The two tasks were executed separately (two sets of CAMRAD II runs). Performance and loads calculations in Ref. [2] and [5] were performed for the 699 rotor with flexible blades and hub, including the gimbal, but with no fixed system flexibility. For the stability calculations (reported in full here and in part previously in Ref. [2]), the fixed system dynamics are represented by analytical NASTRAN modes and by experimental modes, with the same rotor model as for the performance and loads task.

\section{A. 699 rotor model}

The most recent CAMRAD II structural model for the 699 rotor/hub/blade is used in this study. Similar to the V22 CAMRAD model (Ref. [11]), a dual load-path model is used for the 699 rotor. The yoke (flexbeam) and the blade form the two load paths. The CAMRAD II aerodynamic model requires airfoil tables and these were provided by Bell Helicopter as C81 tables. For the performance and loads predictions (Refs. [2], [5]) the CAMRAD II rolled up wake model is used, and for the present stability analyses, the uniform inflow model is used.

The rotor model includes the gimbal and swashplate degrees of freedom. In the trim calculations, for both tasks 
(performance and loads and stability) in the current application, 12 elastic blade modes are used (torsion, flap and lag); for stability analyses, 8 elastic blade modes are used. Table 1 summarizes the modes used for different analyses.

Table 1. Summary of analytical models, adapted from Ref. [2].

\begin{tabular}{|c|c|c|c|}
\hline & \multicolumn{3}{|c|}{ Analysis task } \\
\hline & \multirow[t]{2}{*}{ Performance \& loads } & \multicolumn{2}{|c|}{ Stability } \\
\hline & & Trim & Flutter \\
\hline \multicolumn{4}{|l|}{ Rotor Blade } \\
\hline \multicolumn{4}{|l|}{ Torsion, flap, lag } \\
\hline Total \# of modes per blade & 12 & 12 & 8 \\
\hline \multicolumn{4}{|l|}{ Airframe (TTR) } \\
\hline Drive train, elastic & No & No & Yes \\
\hline Elastic modes, shake test & No & No & $7^{\mathrm{a}}, 8^{\mathrm{b}}$ \\
\hline Elastic modes, NASTRAN & No & No & 15 \\
\hline
\end{tabular}

a 7 TTR modes for 45-deg yaw and 90-deg yaw conditions

b 8 TTR modes for 0 -deg yaw condition

\section{B. Airframe (TTR) model}

Three orientations of the TTR were considered for the stability calculations:

- $\quad 0$-deg yaw (airplane mode)

- 45-deg yaw (conversion)

- $\quad$ 90-deg yaw (helicopter mode)

The 0 -deg yaw condition involves the highest airspeeds and is the most important condition for stability.

For the TTR installed in the NFAC 40x80 test section, shake test modal data are available, Ref. [1]. These experimental modal data are used in the current study. A NASTRAN model of the TTR (discussed later) was used for design and preliminary evaluations of TTR/699 stability, and selected results from these initial studies are also included in this paper. The NASTRAN model was updated to include findings of the shake test.

The stability analyses that are based on experimental data use all available data; that is, 8 elastic modes for the 0 deg yaw airplane condition and 7 elastic modes for the 45-deg yaw conversion and the 90-deg yaw helicopter conditions. The NASTRAN data-based stability analyses use 15 elastic TTR modes. The TTR mode "Nose Yaw" involving lateral motion of the TTR nose has the lowest frequency (approximately $2 \mathrm{~Hz}$ ) and is the most important mode for whirl flutter stability.

\section{NASTRAN Models of the TTR}

The history of the TTR NASTRAN model is summarized here, focusing on changes made subsequent to the original Bell analysis in order to better represent the installation of the TTR in the NFAC 40x80 test section is covered here briefly. Figure 2 is an illustration of the TTR installed in the 40x80 wind tunnel test section.

The purpose of the TTR NASTRAN model is threefold: to ensure there are no excessive structural loads, to ensure there are no dynamic modes too close to a rotor frequency or its $\mathrm{N} / \mathrm{rev}$ multiple, and to provide modal inputs to stability analyses such as CAMRAD II. The third reason is of primary relevance to this study.

The TTR NASTRAN model is constructed from two independently developed models: a model of the TTR itself, developed by Bell Helicopter, and a model of the 40x80 T-frame and supporting structure, developed by NASA. The models are joined by elements representing the $40 \times 80$ mounting struts.

A ground vibration test (GVT- covered in a later section) generated experimental modal data, but in less detail than the NASTRAN model. Selected results from Ref. [1] are included here. Shake test data are generally more accurate than analytical results but are available too late to affect system design, so in any case, a good NASTRAN model remains essential for test planning. 


\section{A. Brief discussion of modifications}

The forward strut of the TTR is ordinarily the 40x80 tail strut. The T-frame was modified to allow 360 -deg rotation to point the rotor either upstream or downstream, or any other angle as appropriate for the conversion mode. These modifications have no effect on the NASTRAN model, and are mentioned here only to point out the non-standard orientation of the rig and tail strut.

The Bell NASTRAN model used the old 40x80 tail strut traversing mount. The 40x80 test section T-frame was subsequently modified by replacing the tail mount with the new "HIADS" fixed mount, which is much stiffer than the older traversing mount. The modifications were made to improve whirl-mode stability, which involves considerable flexing of the forward strut. The TTR rotor extends well beyond the forward strut to provide space for a wing (if needed in future tests). The large overhang causes strong coupling between yaw and lateral modes, plus large in-plane rotor motions for small yaw deflections. These two effects reduce whirl-mode stability, so a stiffer strut mount was needed to reduce flexibility. (The new mount was also needed for a completely separate test, the HIADS test, hence the name.) The NASTRAN model was updated to include the new strut carriage.

Immediately prior to the installation of the TTR in the test section, the mounting struts were load-tested to calibrate their strain gages. Photogrammetry measurements of strut deflections were taken simultaneously, but only for the aft strut pair, and only in the lateral direction. The data revealed that the struts deflected much more at the base than predicted, which implied that the NASTRAN model of the strut attachments was inadequate. The NASTRAN model was modified in February 2018 to better predict the deflections of the struts under load. Further adjustments to the NASTRAN model were made to reflect the as-built weight and c.g., based on measured weights of individual components and of the entire rig.

As noted above, the most important use of the NASTRAN models is to provide inputs to aeroelastic stability analyses and secondarily, to predict $\mathrm{N} / \mathrm{rev}$ frequency crossings. Numerous pre-test predictions of whirl-mode flutter have been made by NASA as the NASTRAN model evolved. The stability predictions of January 2018 (Ref. [2]) are based on experimental modal data (Ref. [1]); the present paper updates the predictions of Ref. [2] to include measured worst-case TTR damping, and also enlarges the operational envelope considered for the stability analysis by considering high thrust levels for the important 90-deg yaw helicopter mode. Thus, this paper contains the complete set of stability results.

\section{B. Selected NASTRAN results}

NASTRAN predictions were obtained for the following TTR orientations: 0-deg yaw (hover and cruise), 45-deg yaw (conversion), and 90-deg yaw (helicopter mode). Examples are given in Table 2, which lists the first eight predicted modal frequencies, and adds the frequencies measured during the shake test (GVT) for low-amplitude random excitation at 0 -deg yaw. For the important nose yaw mode (lowest frequency mode in the table), the latest NASTRAN model compares favorably with the experimental value.

Sample stability results obtained using the NASTRAN modes of January/February 2017 are shown later in the Results section, along with the definitive worst-case damping stability results obtained using the experimental modes. 
Table 2. Sample of NASTRAN frequency changes, 0-deg yaw (airplane mode).

\begin{tabular}{|c|c|c|c|}
\hline \multirow[t]{2}{*}{ Mode } & \multicolumn{3}{|c|}{ Frequency, $\mathrm{Hz}$} \\
\hline & NASTRAN & NASTRAN & $\underline{\mathrm{GVT}}$ \\
\hline & July 2017 & Feb 2018 & (shake test, Ref. [1]) \\
\hline Rigid-body Yaw ("Nose Yaw") & 2.28 & 2.07 & 1.97 \\
\hline Rigid-body Axial (“RB Axial”) & 3.23 & 2.93 & 2.58 \\
\hline Rigid-body Lateral (“CG Yaw”) & 3.89 & 3.22 & 2.59 \\
\hline T-Frame Vertical & 9.02 & 8.99 & 8.40 \\
\hline Rotor Mast Vertical & 11.25 & 11.22 & 11.4 \\
\hline T-Frame Axial & 12.13 & 12.18 & \\
\hline T-Frame Yaw & 12.81 & 12.68 & \\
\hline Rotor Mast Lateral & 13.21 & 12.90 & 14.8 \\
\hline
\end{tabular}

\section{Experimental Efforts: TTR Shake and Impact Testing}

The modal test procedure, modal test results, and worst-case modal data are covered in this section. Here, worstcase refers to modal data that has the lowest level of measured damping. The current stability calculations that are based on test modal data use these worst-case data.

\section{A. Modal test description}

The modal characteristics of the TTR were determined primarily by shake testing, Ref. [1]. Impact (rap) testing was also used, both to verify the results of the shake test, and to determine whether impact testing could be used in lieu of shake testing for future tests. The shake test setup for longitudinal shaking is shown in Fig. 3. Excitation loads were applied in all three principal axes of the TTR. The setup consisted of a hydraulic shaker backstopped against a $12,000-1 \mathrm{~b}$ reaction mass and secured to the wind tunnel floor by guywires. A load cell measured the input loading, and the input waveform was generated by the data acquisition system.

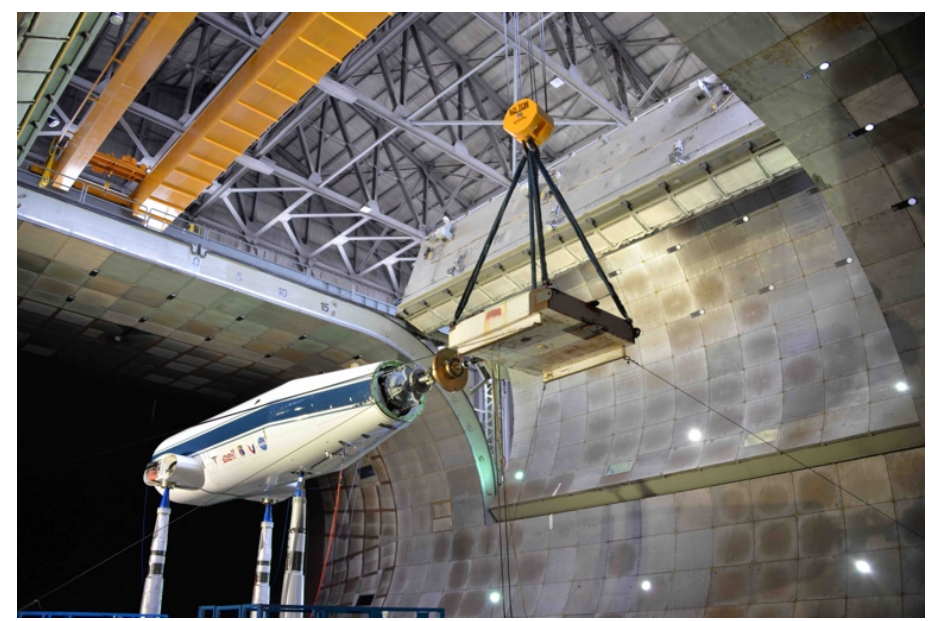

Fig. 3 Shake test setup - longitudinal shaking at hub - 0-deg yaw (airplane mode).

With the system described above, input loads of up to $\pm 1,000 \mathrm{lb}$ with arbitrary waveforms and frequency content up to $40 \mathrm{~Hz}$ and amplitude were possible. The two types of waveform used for this shake test were random and swept sine. Once frequency ranges of interest were identified by the random excitation, swept sine excitation was used to further characterize the vibratory response of the TTR in these narrow frequency bands. Compared to random excitation, swept sine excitation applies more energy at each frequency, but presents a greater challenge for maintaining good load alignment. 
The impact testing was carried out in order to determine whether future modal testing of the TTR could be accomplished without the need for a time-consuming shake test. The complete shake test, described in Ref. [1], included 16 combinations of load application and yaw angle. It took over a month to complete, due to installation of the shake test hardware in the various required locations and directions on the TTR. An equivalent impact test could likely be completed in a week. For the test described here, the impact testing was conducted for all of the same directions and locations as the shake test. In addition, the impact test included excitation with the TTR positioned at 45-deg yaw.

\section{B. Modal test results}

This section presents a brief overview of the modal test results. Results were obtained for excitation at three different locations, including the hub, forward lifting lug, and the port aft lifting lug. Excitation at the lifting lugs allowed the input loads to bypass the rotor hub and directly excite the TTR. Mode shapes along with frequency and damping values were obtained for eight different modes within the $0-40 \mathrm{~Hz}$ frequency range of interest. For 0-deg yaw, these modes are summarized in Table 3 below (and also included in Table 2 in the "GVT" column). The descriptive labels for the modes in Table 3 are slightly different from those used in Table 2; the labels within quotation marks are important for stability and common to both tables. The first four modes are easy to identify in both the NASTRAN and GVT data. At high frequencies, NASTRAN gives more detailed mode shapes but is less accurate in frequency; the mode labels reflect this difference.

Table 3. Experimental TTR modal frequency and damping, 0-deg yaw (airplane mode), random excitation at hub, Ref. [1].

\begin{tabular}{cccl}
\hline Mode & Frequency, Hz & Damping, \% critical & \multicolumn{1}{c}{ Description } \\
\hline 1. & 1.97 & 2.97 & Rigid body yaw mode about aft struts ("Nose Yaw') \\
2. & 2.58 & 0.93 & Rigid body longitudinal strut mode ("RB Axial") \\
3. & 2.59 & 1.48 & Rigid body yaw mode about fwd strut ("CG Yaw") \\
4. & 8.40 & 1.45 & T-frame vertical mode \\
5. & 11.4 & 0.83 & TTR + T-frame pitch \\
6. & 14.8 & 0.84 & Lateral mast bending \\
7. & 15.3 & 2.07 & Vertical mast + TTR bending \\
8. & 17.1 & 1.22 & TTR + vertical mast bending \\
\hline
\end{tabular}

The low-frequency strut modes are of primary interest for the stability calculations, as they are the modes that can contribute to whirl flutter instabilities. Figure 4 shows the scatter in damping values obtained for different excitation methods and locations at various TTR yaw angles. In order to determine the worst-case scenario, the lowest damping values obtained were used for the stability analyses (Results section). Because of the difficulties with swept sine testing mentioned above, there was not a high level of confidence in the damping values obtained from the swept sine method.

\section{Worst-case damping modal data}

The worst-case damping was selected from results for random and impact excitation. The resulting frequency and damping sets are listed in Tables 4-6 for the three TTR orientations of interest: 0-deg, 45-deg, and 90-deg yaw, respectively. Unless noted otherwise, the stability analyses presented in this paper are based on the worst-case modal data given in Tables 4-6, and hence represent updated, worst-case stability calculations compared to those presented earlier in Ref. [2]. 


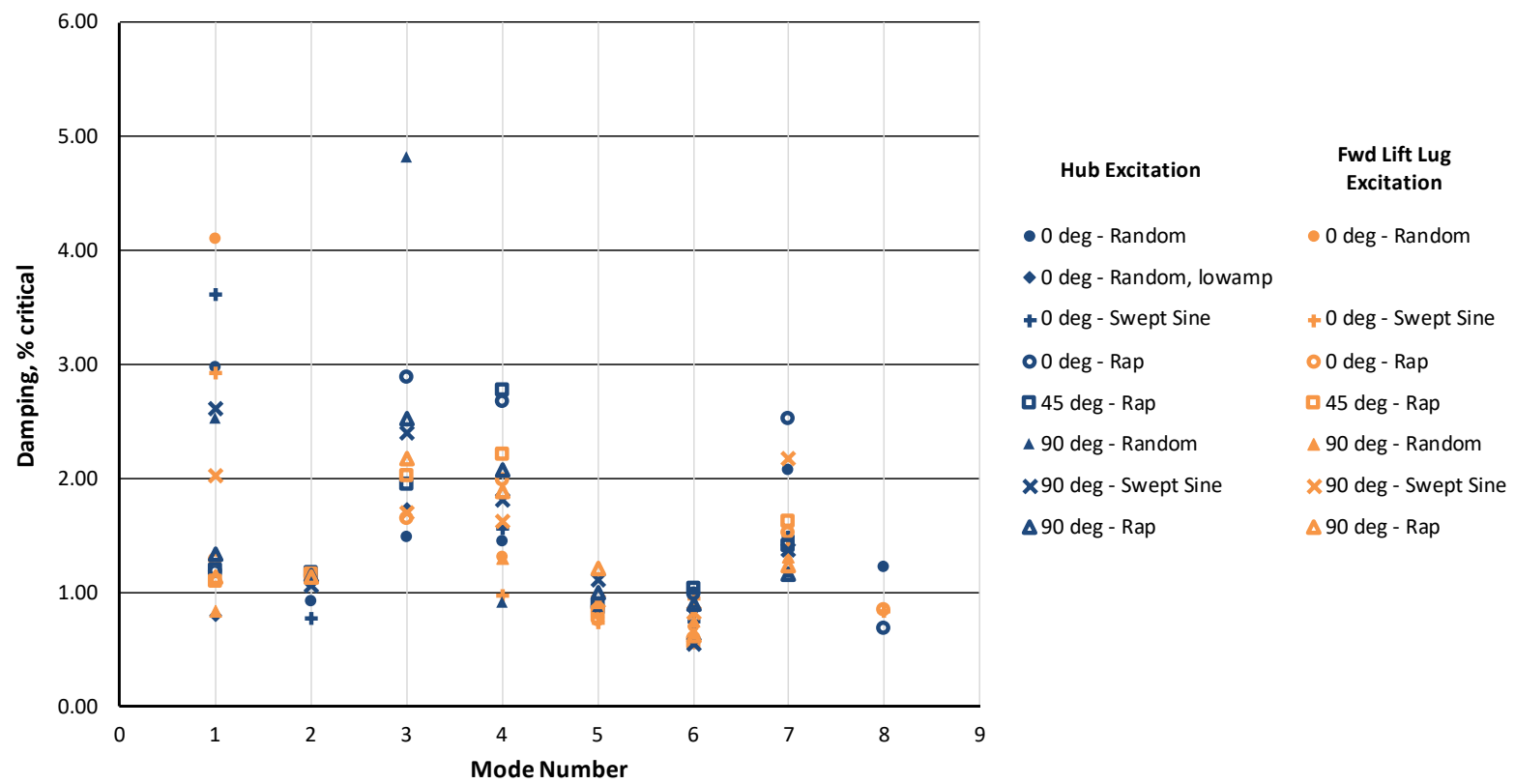

Fig. 4 Comparison of modal damping values - hub and forward (fwd) lifting lug.

Table 4. 0-deg yaw worst-case TTR mode frequency and damping (Ref. [1]).

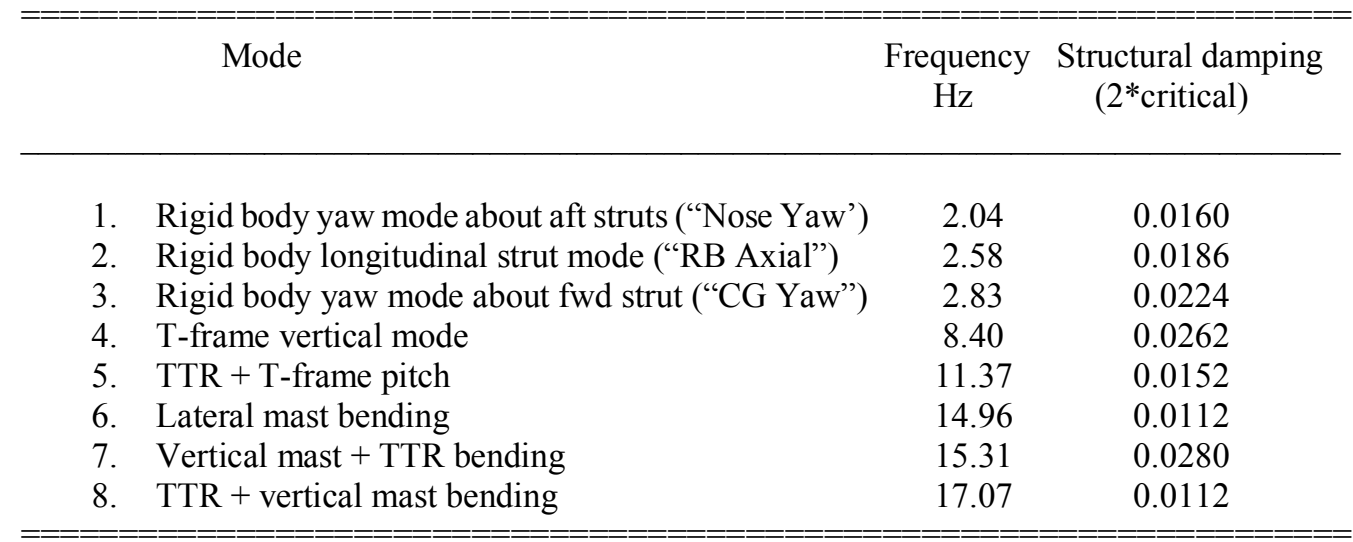

Table 5. 45-deg yaw worst-case TTR mode frequency and damping (Ref. [1]).

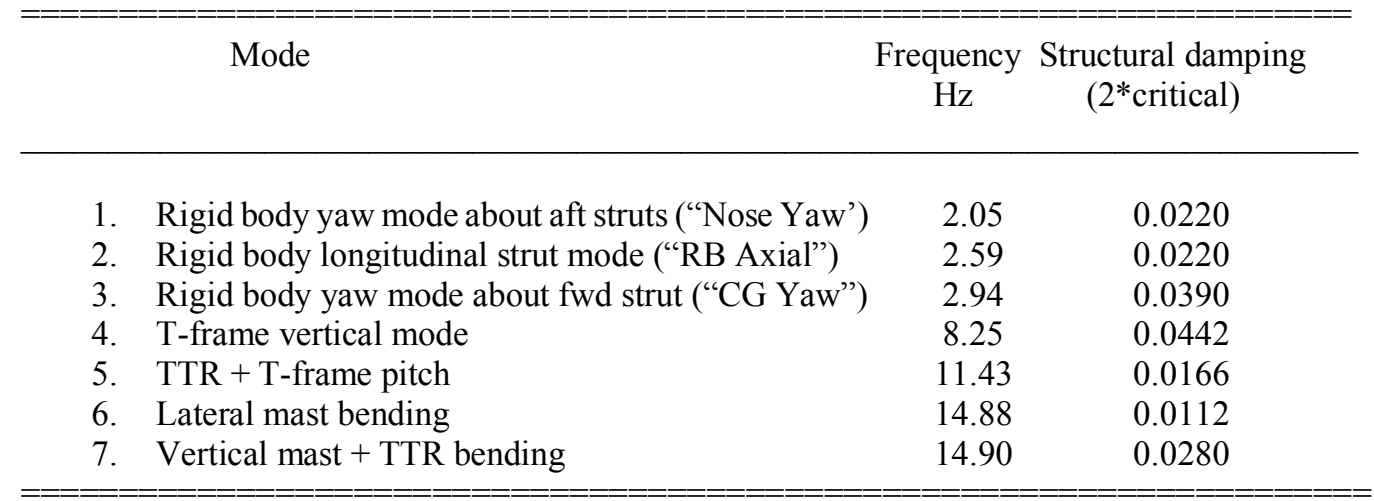


Table 6. 90-deg yaw worst-case TTR mode frequency and damping (Ref. [1]).

\begin{tabular}{|c|c|c|}
\hline Mode & Frequency & Structural damping \\
\hline 1. Rigid body yaw mode about aft struts ("Nose Yaw') & 2.06 & 0.0166 \\
\hline 2. Rigid body longitudinal strut mode ("RB Axial") & 2.52 & 0.0222 \\
\hline 3. Rigid body yaw mode about fwd strut ("CG Yaw") & 2.79 & 0.0434 \\
\hline 4. T-frame vertical mode & 8.40 & 0.0184 \\
\hline 5. TTR + T-frame pitch & 11.47 & 0.0176 \\
\hline 6. Lateral mast bending & 14.94 & 0.0110 \\
\hline 7. Vertical mast + TTR bending & 14.72 & 0.0230 \\
\hline
\end{tabular}

\section{Results}

The results presented in this paper represent the stability part of all the analytical results that were obtained for the TTR test. The analytical studies include performance and loads predictions in addition to the stability predictions. As background, to give an idea of the scope of the full set of analytical studies, Fig. 5 from Ref. [2] shows the 699 approximate conversion corridor and the conditions at which the performance, loads and stability calculations were performed (the conversion corridor is derived from Refs. [12] and [13]). Note that the analysis points in Fig. 5 refer to performance and loads calculations; the stability calculations were performed at the following three TTR orientations (nacelle angles): 0-deg, 45-deg, and 90-deg yaw, discussed as follows.

Stability calculations for the TTR/699 installed in the 40x80 wind tunnel for hover, airplane (0-deg yaw), conversion, and helicopter modes (45-deg and 90-deg yaw, respectively) are shown in the following sections. In this paper, experimental modal data-based predictions are given emphasis. Selected NASTRAN data-based predictions are also included here to demonstrate rough corroboration.

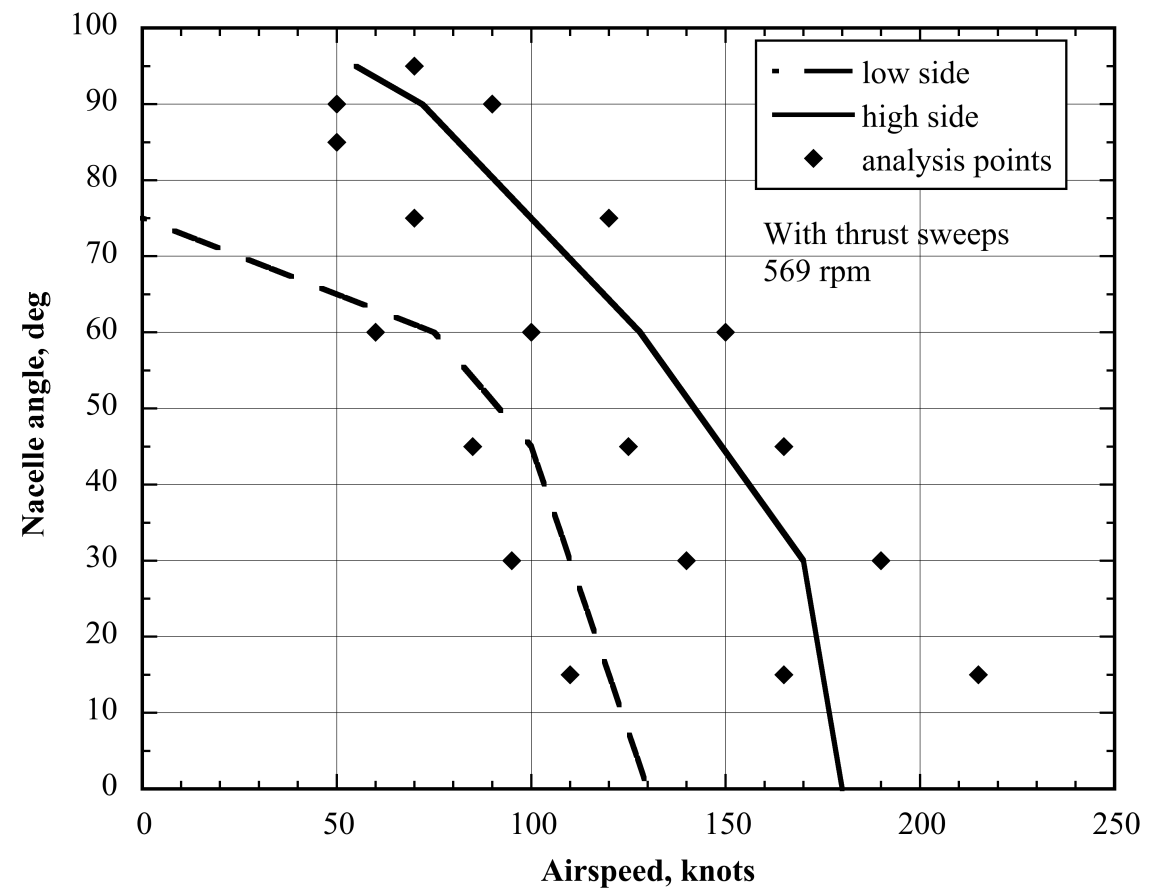

Fig. 5. Approximate AW609 conversion corridor (Refs. [12] and [13]) - analysis points refer to performance and loads calculations, not current stability calculations (see text), figure is from Ref.[2]. 


\section{A. Hover}

Figures $6 \mathrm{a}-6 \mathrm{~b}$ show worst-case hover modal damping and frequency variations vs. $\mathrm{C}_{\mathrm{T}} / \sigma$, at $569 \mathrm{rpm}$. To elaborate on the modes shown in the figures, the CAMRAD II stability model used a semi-flexible drive-train (Table 1), where the motors and gearbox were rigidly coupled together, but the rotor was coupled to the gearbox with a torsionally flexible shaft. The rotor is coupled to the gearbox with a segmented drive train, including an inner drive shaft and an outer rotor mast connected by a gear coupling. Herein "shaft (lag)" mode refers to the first drive shaft torsional mode coupled with the first rotor lag mode, and "mast" modes are transverse bending modes of the rotor mast. In Figs. 6a$6 \mathrm{~b}$, the mast mode is primarily the lateral bending mode, but with heavy participation of the vertical mast and T-frame pitch modes. Figure $6 \mathrm{a}$ shows that the rotor is completely stable up to at least $\mathrm{C}_{\mathrm{T}} / \sigma=0.2$, which approximately equals $15,000 \mathrm{lb}$ thrust and requires $2719 \mathrm{hp}$. In the current checkout test, the TTR was operated with two motors - the power limit is $2500 \mathrm{hp}$. Hover stability is therefore of no concern for the TTR/699 with only two motors operating. Note that the instability encountered at high thrust in hover is associated with a loss of flap damping when the rotor stalls $\left(\mathrm{C}_{\mathrm{T}} / \sigma \geq 0.225\right.$, Fig. 6a).

\section{B. Cruise (airplane mode)}

In axial flow (airplane mode), zero power is the worst case for whirl flutter for a gimballed rotor with precone. Figures $7 \mathrm{a}-7 \mathrm{~b}$ show worst-case cruise damping and frequency variations vs. airspeed for zero power, $478 \mathrm{rpm}$ Figure 6a shows that the TTR/699 in the NFAC 40x80 wind tunnel is stable with close to 1\% critical damping for airspeeds $<300$ knots (the maximum wind tunnel airspeed). The critical mode for stability is the nose yaw mode, which involves lateral bending of the forward support strut (Figs. 1-2). This mode is specific to the TTR as installed in the 40- by 80$\mathrm{ft}$ test section. See Ref. [13] for a discussion of aeroelastic stability of the actual aircraft.

\section{Conversion, 45-deg yaw}

For the conversion mode condition that combines edgewise and axial flow, the 699 rotor was trimmed to specified thrust and zero one-per-rev (1P) flapping. For conversion (and helicopter mode) conditions, the wind tunnel test envelope is restricted to airspeeds less than 150 knots based on TTR strut load limits. Stability envelopes based both on NASTRAN modes and experimental modes are shown in this paper.

Compared to the results of Ref. [2], the current predictions involve changes only to the TTR modes (worst-case TTR modes are used here). The rotor model is identical to that of Ref. [2]; that is, the rotor trim procedure and resulting performance parameters (trimmed thrust values, resulting torque and trim cyclics) all remain the same. Thus, Figs. 10a-10d of Ref [2] that show these performance parameters vs. airspeed are completely applicable here and, for completeness, are included in this paper as Figs. 8a-8d. Figure 8a shows the trimmed thrust values for which the stability calculations were performed. The torque (Fig. 8b) is limited by the TTR/699 test limit (static design load limit) and the cyclic control angles (Figs. 8c-8d) are limited by the 699 gimbal angle limits. In these analyses, the rotor is being pushed well outside of the aircraft flight envelope (Fig. 5). The trimmed values of torque and the cyclics therefore represent values not always reachable in flight.

Figures 9a-9b show 45-deg yaw stability envelopes based on NASTRAN and worst-case experimental modal data, respectively. The NASTRAN-based envelope, Fig. 9a, is shown for $0.8 \%$ critical damping and the test-based envelope, Fig. $9 \mathrm{~b}$, is shown for $1 \%$ critical damping. As noted earlier, NASTRAN data-based predictions have been included in this paper to demonstrate rough corroboration with the test data-based predictions, and Figs. 9a-9b serve this purpose. The two different damping values were selected to merely show the similarity of the envelopes - one analytical modal data-based and the other, experimental modal data-based. The test modal data-based stability envelope of Fig. 9b shows that the TTR/699 is predicted to be stable (damping $\geq 1 \%$ critical) within the test envelope for thrust values smaller or equal to $8000 \mathrm{lb}$; for $10,000 \mathrm{lb}$ thrust, comparable stability is obtained up to 100 knots. These stability limits lie beyond what is actually achievable in the wind tunnel; see Ref. [2] for predicted thrust and torque limits vs. airspeed. 


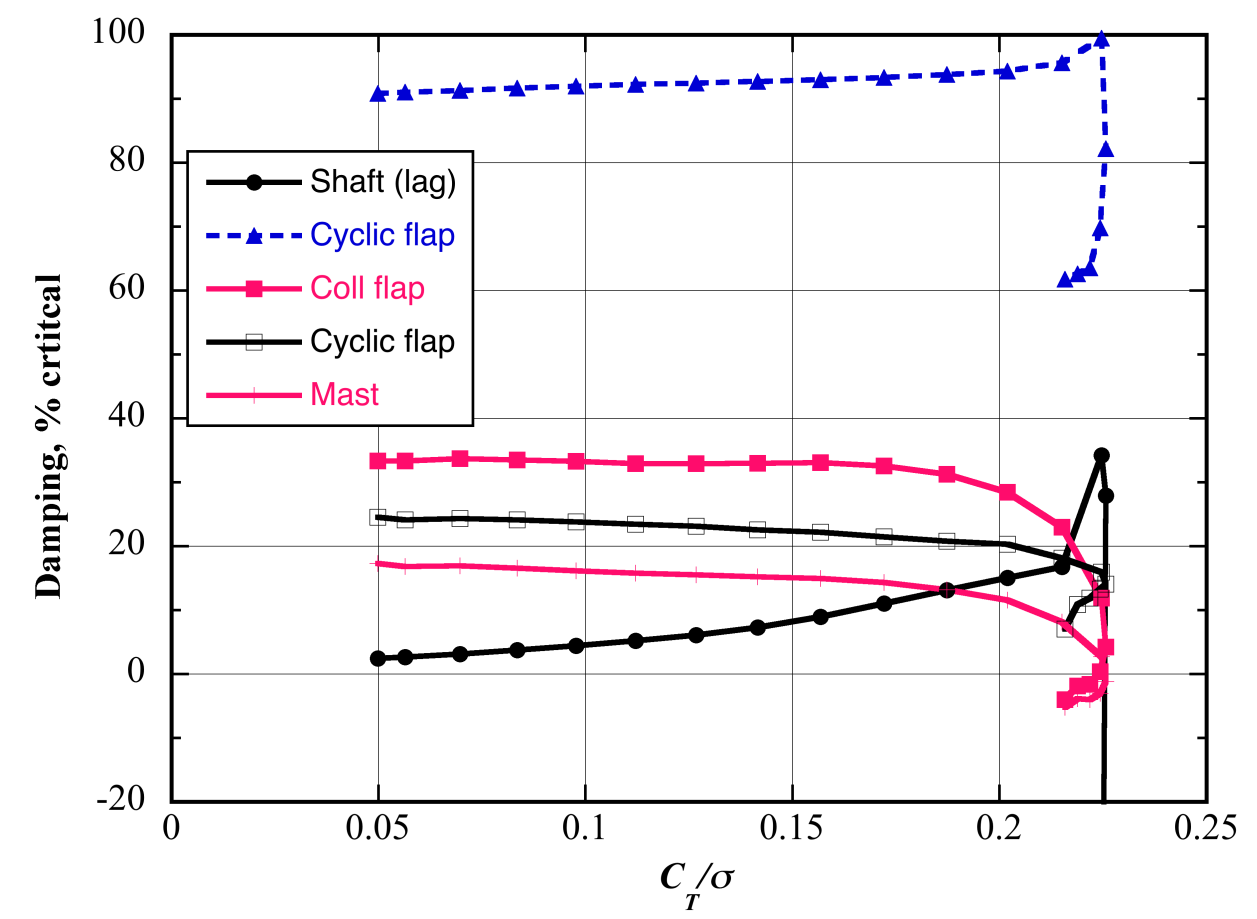

Fig. 6a. Predicted (worst-case) TTR/699 hover damping vs. thrust (0-deg yaw, 569 rpm).

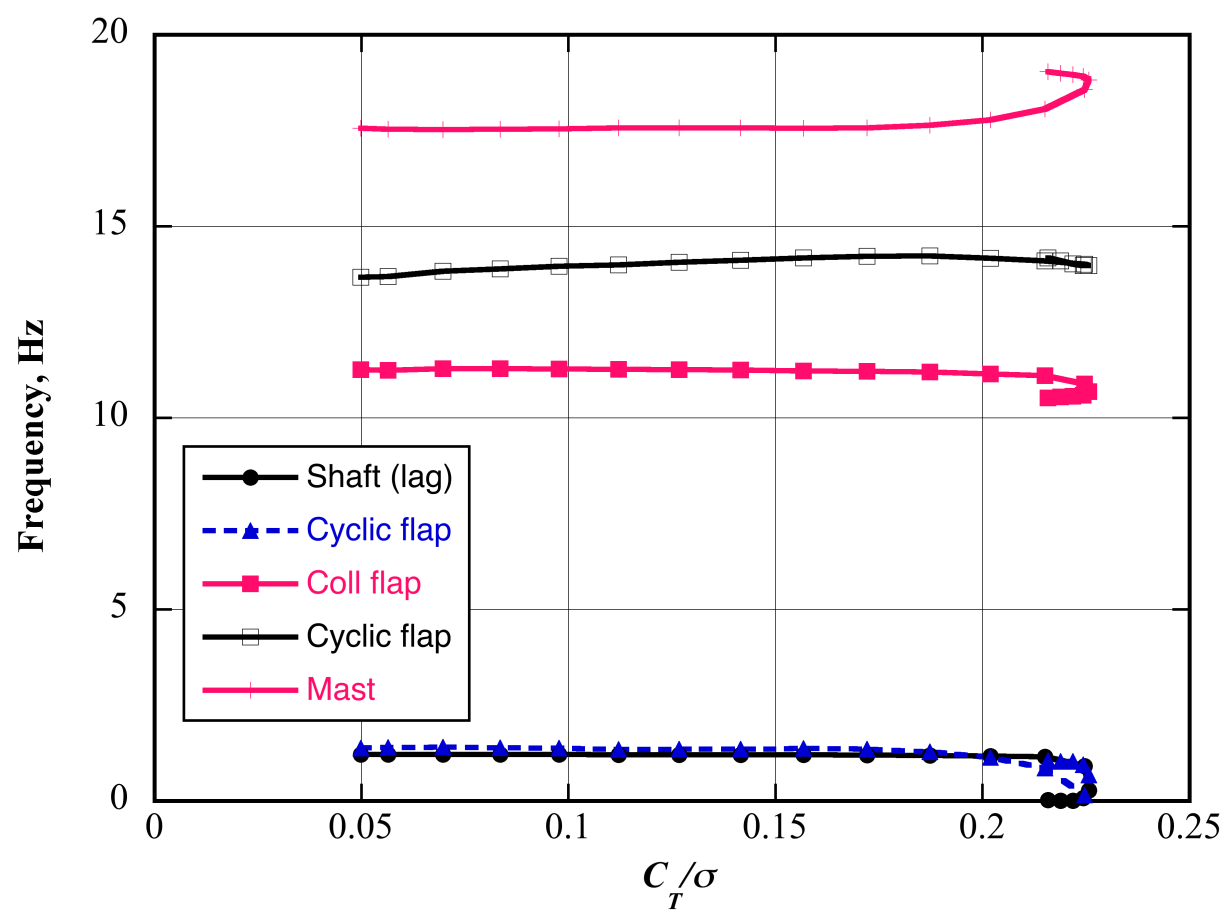

Fig. 6b. Predicted (worst-case) TTR/699 hover frequencies vs. thrust (0-deg yaw, 569 rpm). 


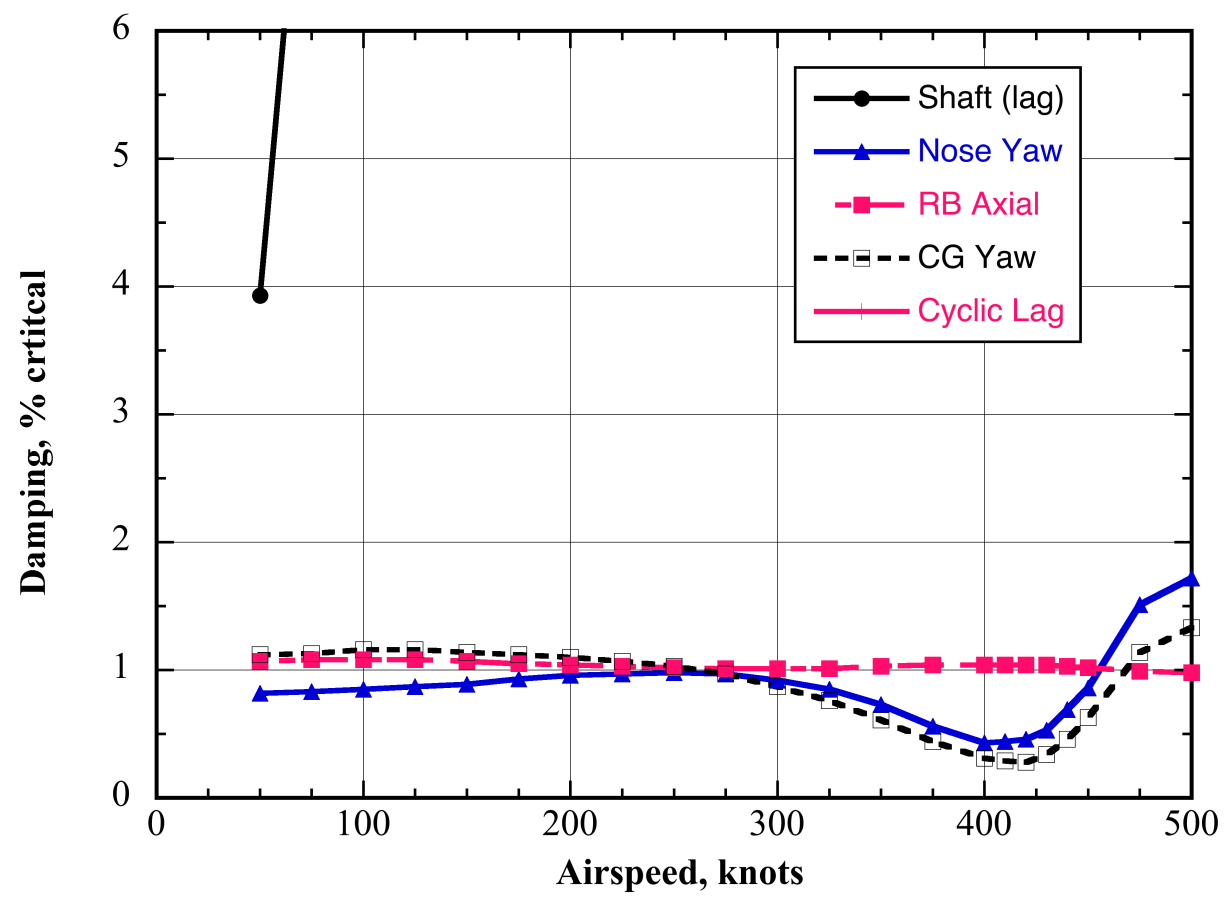

Fig. 7a. Predicted (worst-case) 0 power TTR/699 cruise (axial flow) damping vs. airspeed (0-deg yaw, 478 rpm).

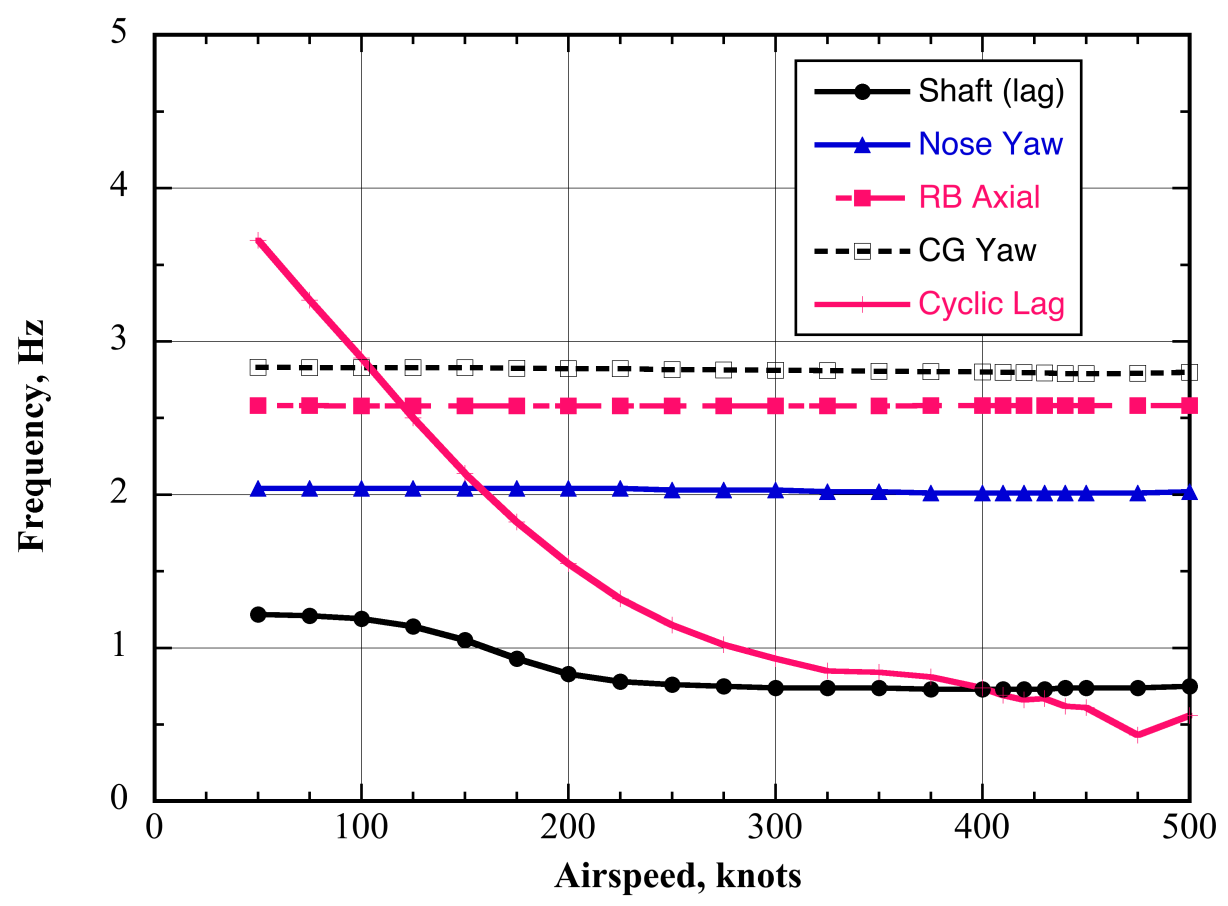

Fig. 7b. Predicted (worst-case) 0 power TTR/699 cruise (axial flow) frequencies vs. airspeed (0-deg yaw, 478 rpm). 


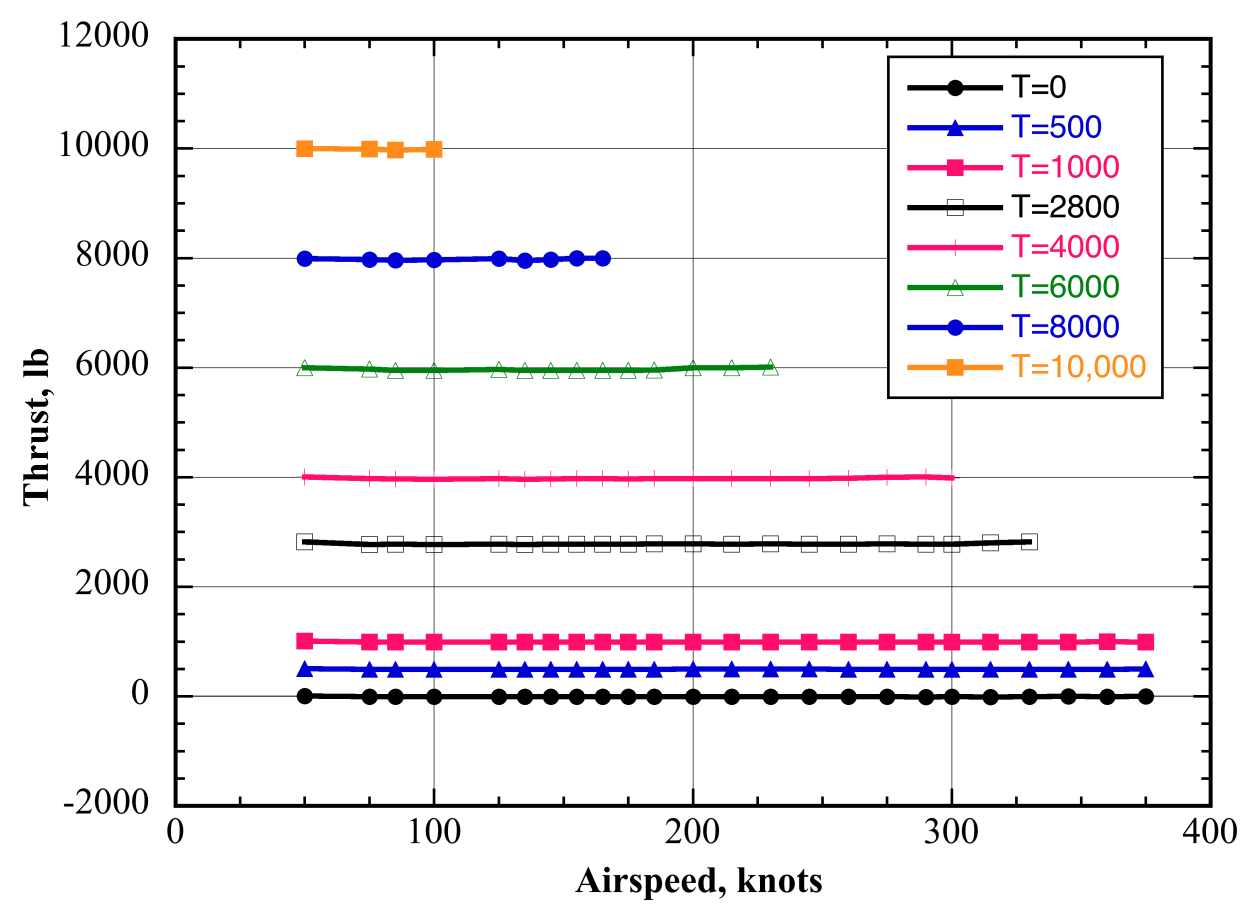

Fig. 8a. Predicted 699 conversion mode trim thrust vs. airspeed (45-deg yaw, 569 rpm).

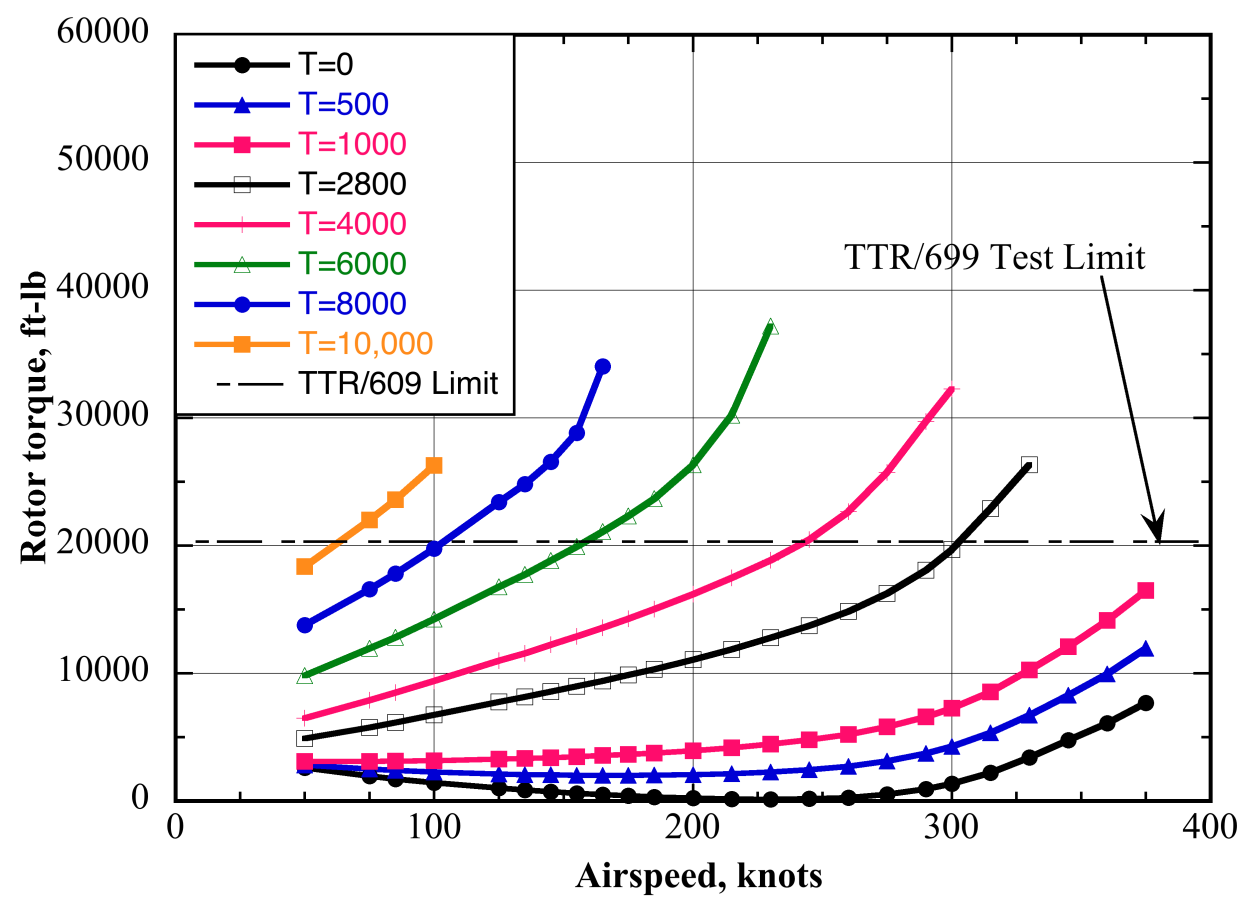

Fig. 8b. Predicted 699 conversion mode torque vs. airspeed (45-deg yaw, 569 rpm). 


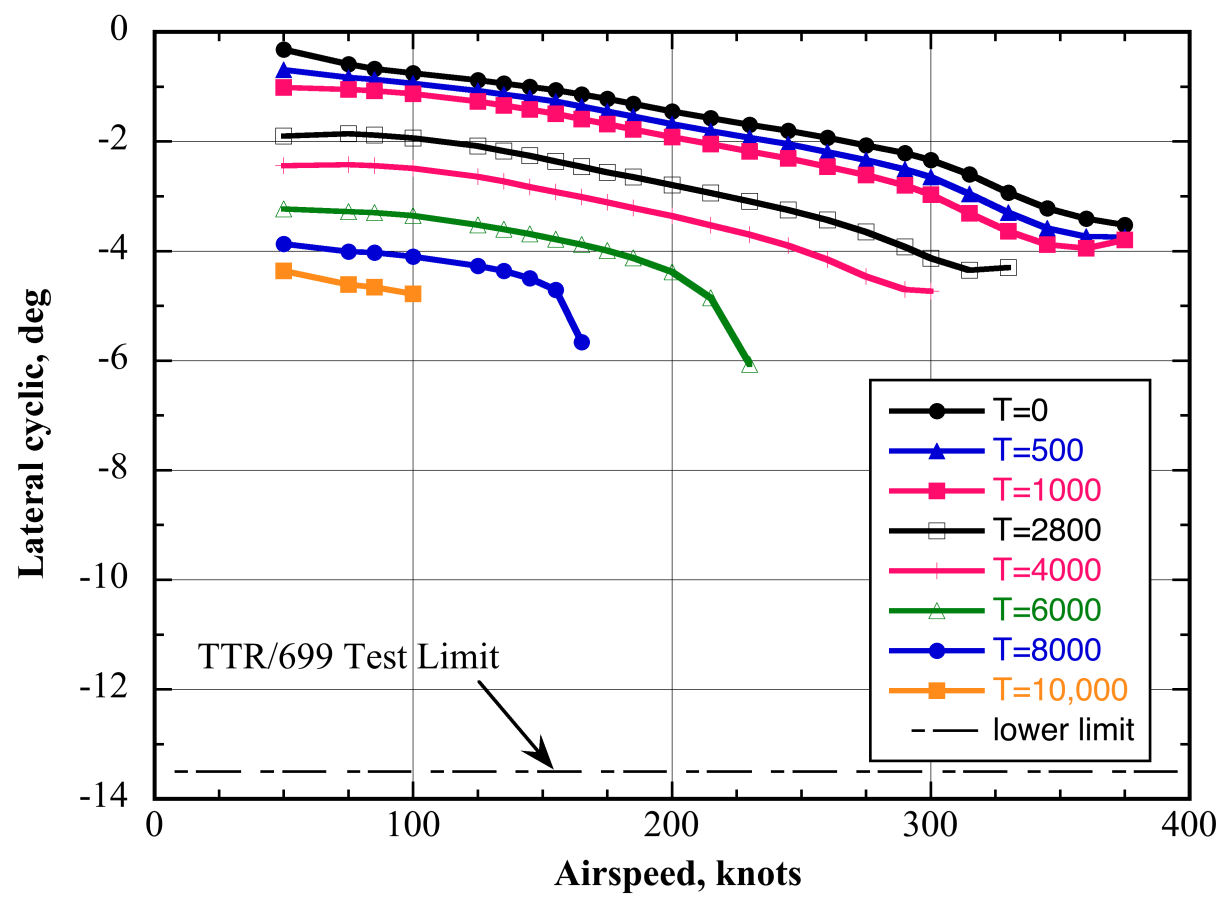

Fig. 8c. Predicted 699 conversion mode lateral cyclic vs. airspeed (45-deg yaw, 569 rpm).

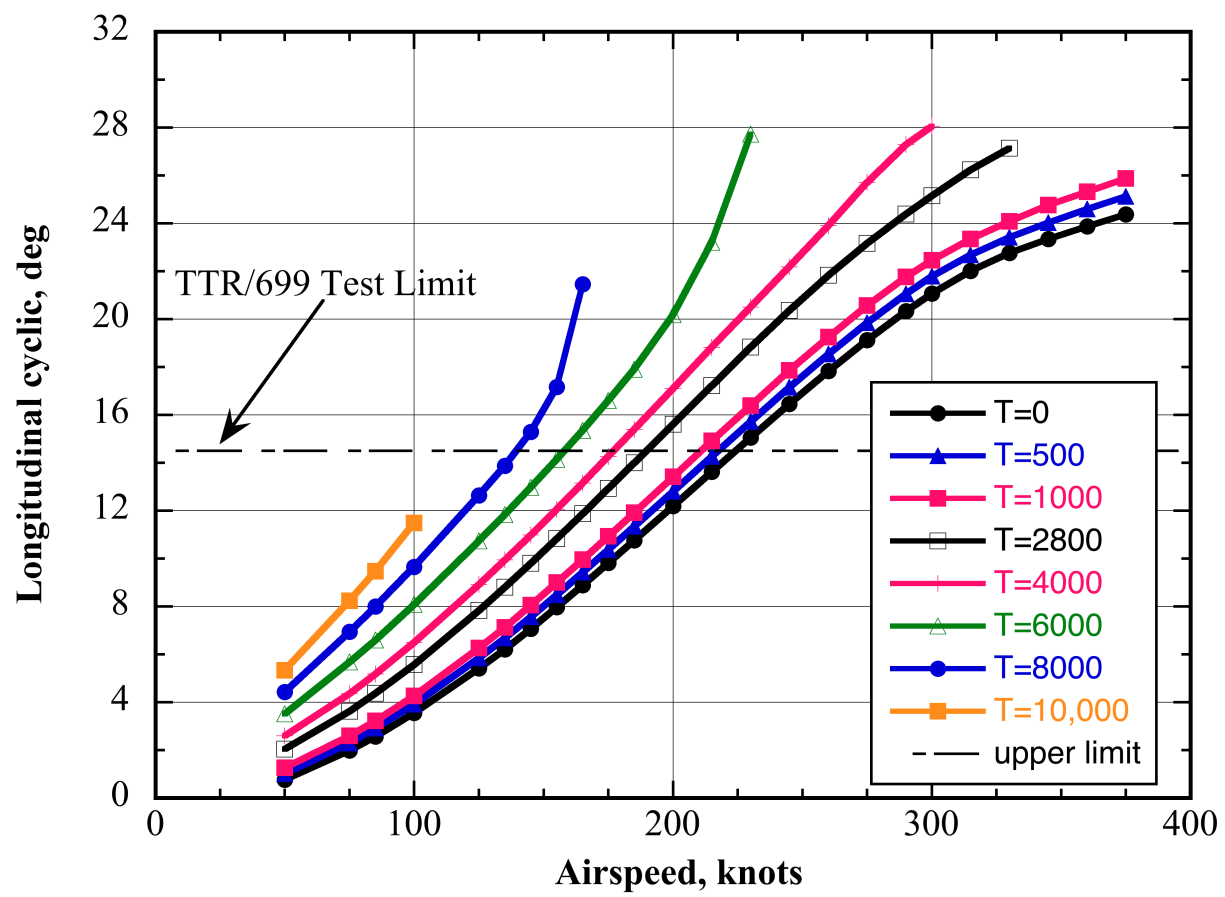

Fig. 8d. Predicted 699 conversion mode longitudinal cyclic vs. airspeed (45-deg yaw, 569 rpm). 


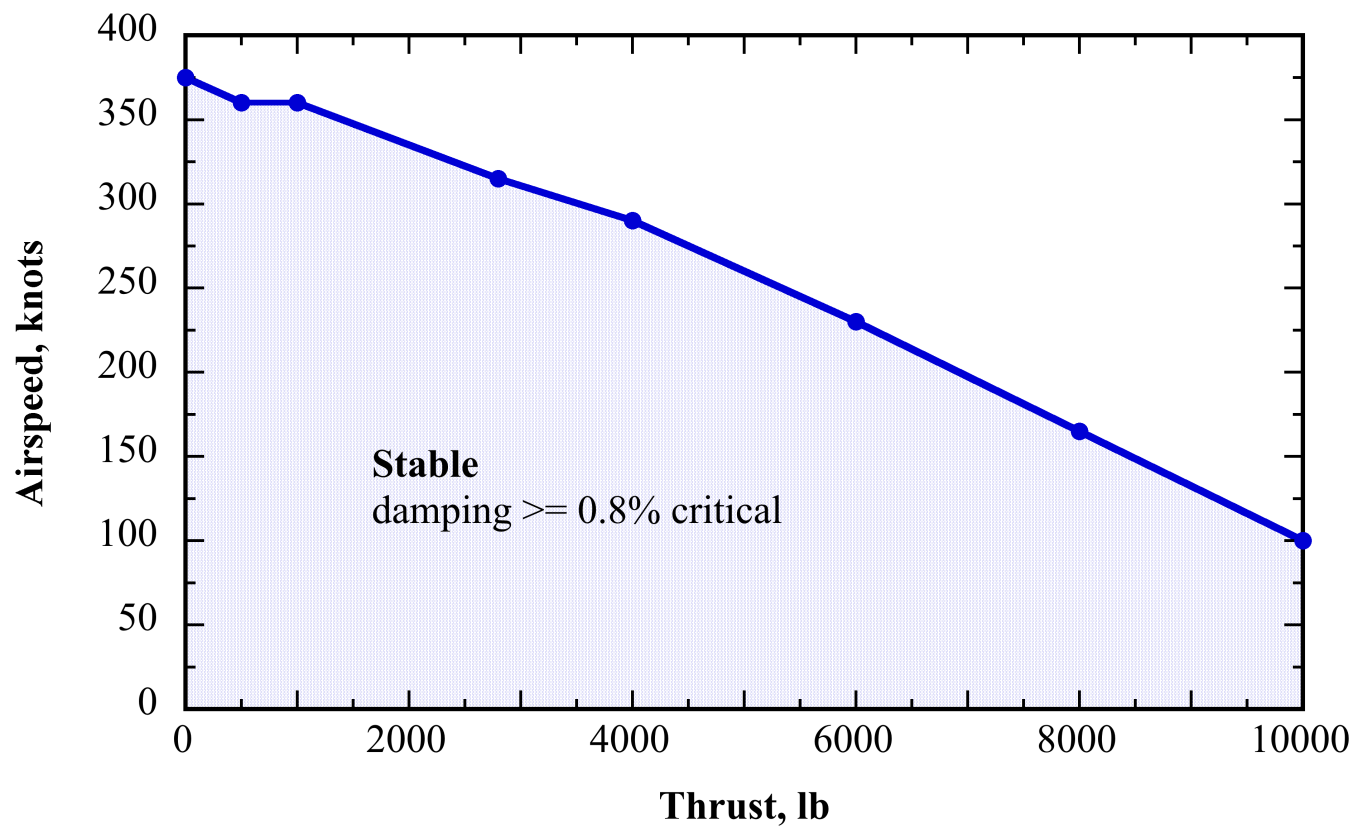

Fig. 9a. Predicted NASTRAN data-based TTR/699 conversion $0.8 \%$ damping stability envelope (45-deg yaw, 569 rpm).

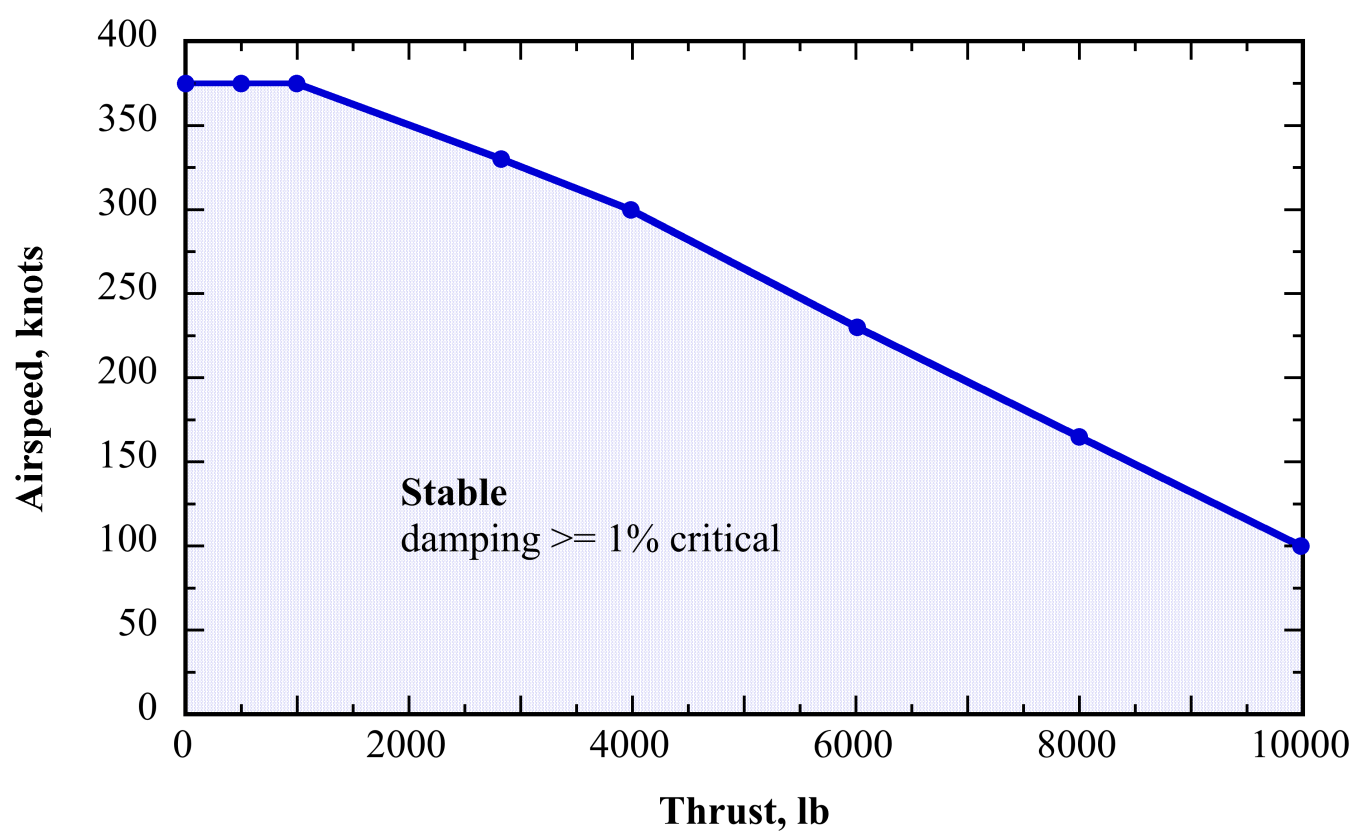

Fig. 9b. Predicted (worst-case) impact test data-based TTR/699 conversion 1\% damping stability envelope (45-deg yaw, 569 rpm).

\section{Helicopter mode, 90-deg yaw}

For this important operational mode, two separate sets of calculations were performed: first, similar to the 45-deg yaw conversion mode, the rotor was trimmed to specified thrust and zero 1P flapping and, second, in order to raise 
the thrust level for the stability calculations, i.e., expand the analytical stability envelope to cover all anticipated test conditions, the rotor was trimmed to zero 1P flapping (with collective sweep, no thrust trim). Also, this is the procedure that is followed during a typical wind tunnel run. These calculations are referred to as "high thrust" calculations.

The results with the rotor trimmed to specified thrust and zero 1P flapping are discussed as follows. Figs. 10a-10c show the 90-deg yaw trim quantities (torque, and lateral and longitudinal cyclics) vs. airspeed for nine thrust values (569 rpm). The torque is shown in Fig. 10a along with the TTR/699 test limit (static design load limit). For the trim cyclics shown in Figs. 10b-10c, the lower and upper limits are also shown; as noted earlier, these TTR/699 test limits are based on the 699 gimbal angle limits. To reiterate, for these analyses, the rotor is being pushed well outside of the aircraft flight envelope (Fig. 5). The trimmed values of torque and cyclic in Figs. 10a-10c therefore represent values not always reachable in flight, and lie beyond the TTR's helicopter-mode airspeed limit for positive thrust.

Figures 11a-11b show 90-deg yaw stability envelopes based on NASTRAN and worst-case experimental modal data, respectively. The NASTRAN-based envelope, Fig. 11a, is shown for $1 \%$ critical damping and the test-based envelope, Fig. $11 \mathrm{~b}$, is shown for $0.7 \%$ critical damping. As noted earlier, NASTRAN data-based predictions have been included in this paper to demonstrate rough corroboration with the test data-based predictions, and Figs. 11a-11b serve this purpose. The two different damping values were selected to merely show the rough similarity of the envelopes - one analytical modal data-based and the other, experimental modal data-based. The test modal data-based stability envelope of Fig. $11 \mathrm{~b}$ shows that the TTR/699 is predicted to be stable (damping $\geq 0.7 \%$ critical) within the test envelope (airspeed $\leq 150$ knots) for thrust values $\leq 9000 \mathrm{lb}$; for $10,000 \mathrm{lb}$ thrust, comparable stability is obtained up to 120 knots.

The 90-deg yaw helicopter mode high thrust results are discussed as follows. Here, the rotor was trimmed to zero 1P flapping (collective sweep, no thrust trim). Figs. 12a-12d show the performance quantities (thrust, torque, and lateral and longitudinal cyclics) vs. collective for nine airspeeds (569 rpm). Where applicable, the TTR/699 test limits are also noted. Figures 13a-13b show the damping and frequency versus collective for the important nose yaw mode of the TTR (frequency approximately $2 \mathrm{~Hz}$ ). Figure 13a shows that the nose yaw goes unstable at higher airspeeds and collective; this instability occurs under extreme conditions, outside the flight envelope, and is not a cause for concern. For completeness, Fig. 14 shows the 1\% damping stability envelope.

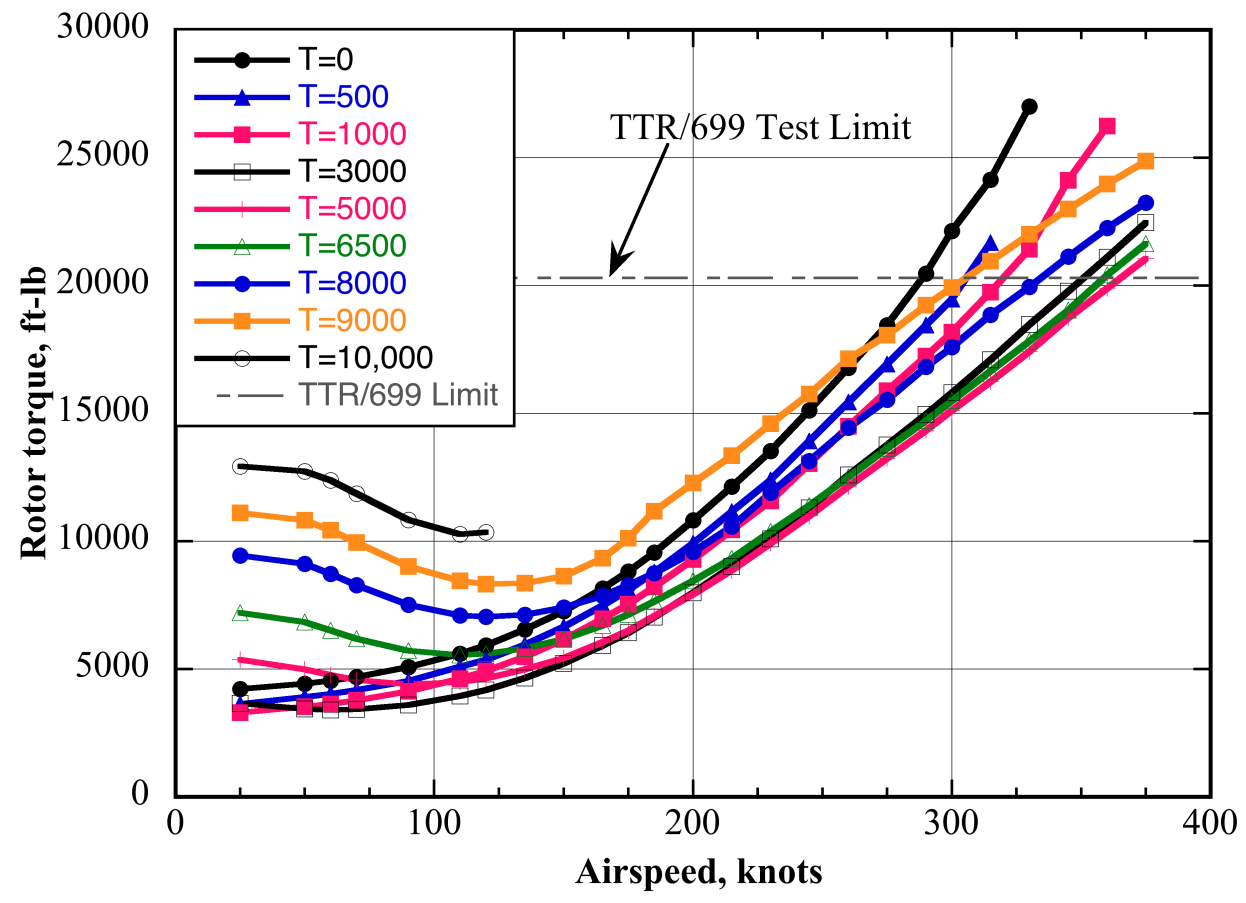

Fig. 10a. Predicted 699 helicopter mode torque vs. airspeed (90-deg yaw, 569 rpm). 


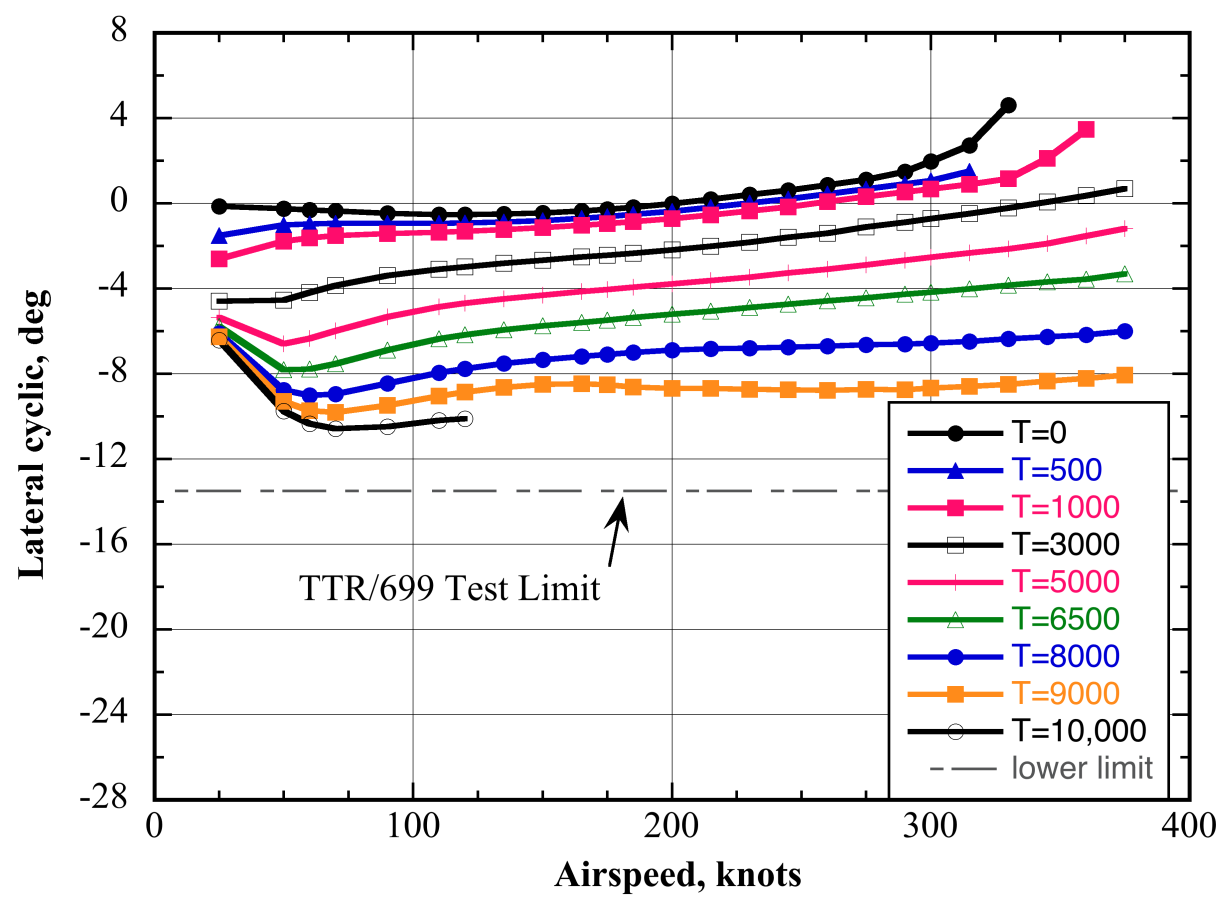

Fig. 10b. Predicted 699 helicopter mode lateral cyclic vs. airspeed (90-deg yaw, 569 rpm).

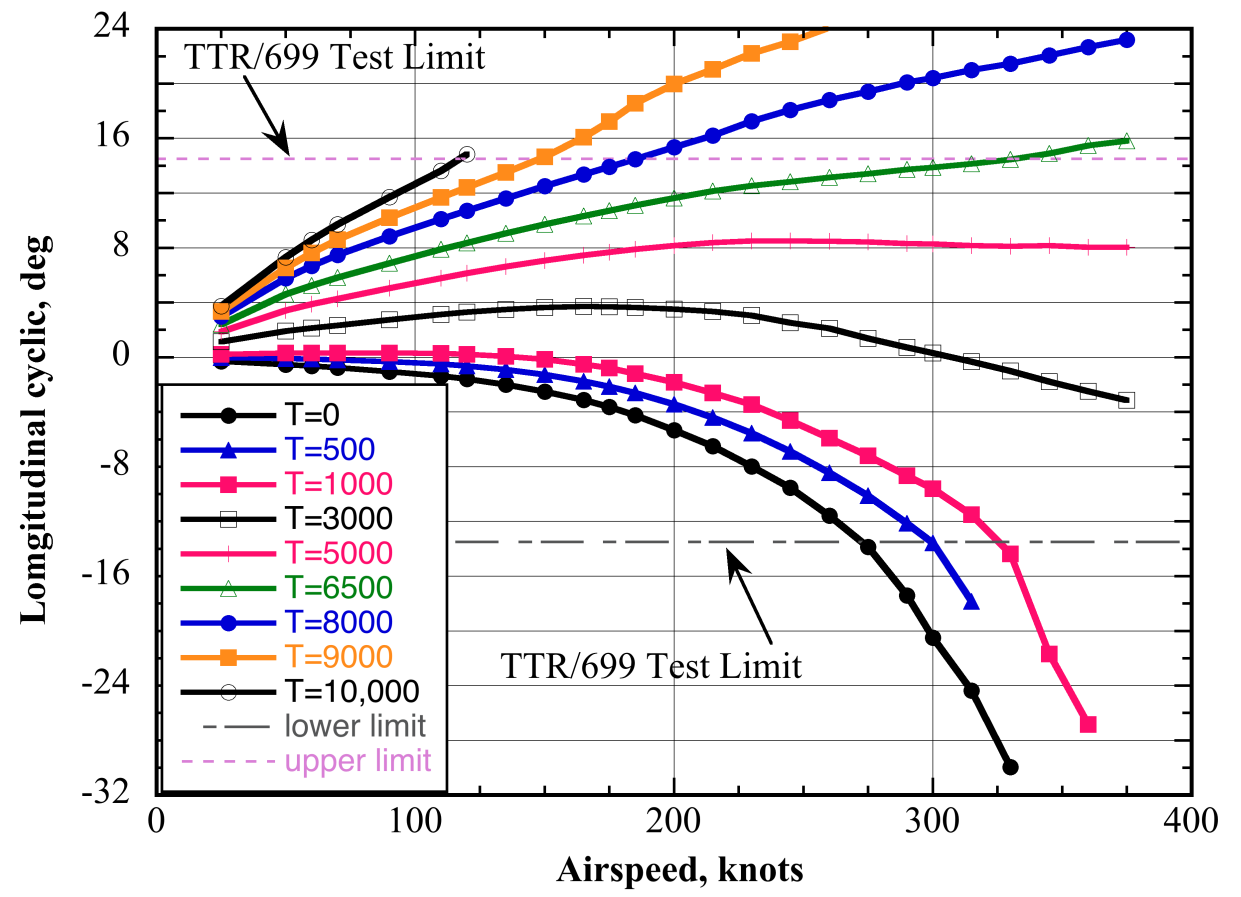

Fig. 10c. Predicted 699 helicopter mode longitudinal cyclic vs. airspeed (90-deg yaw, 569 rpm). 


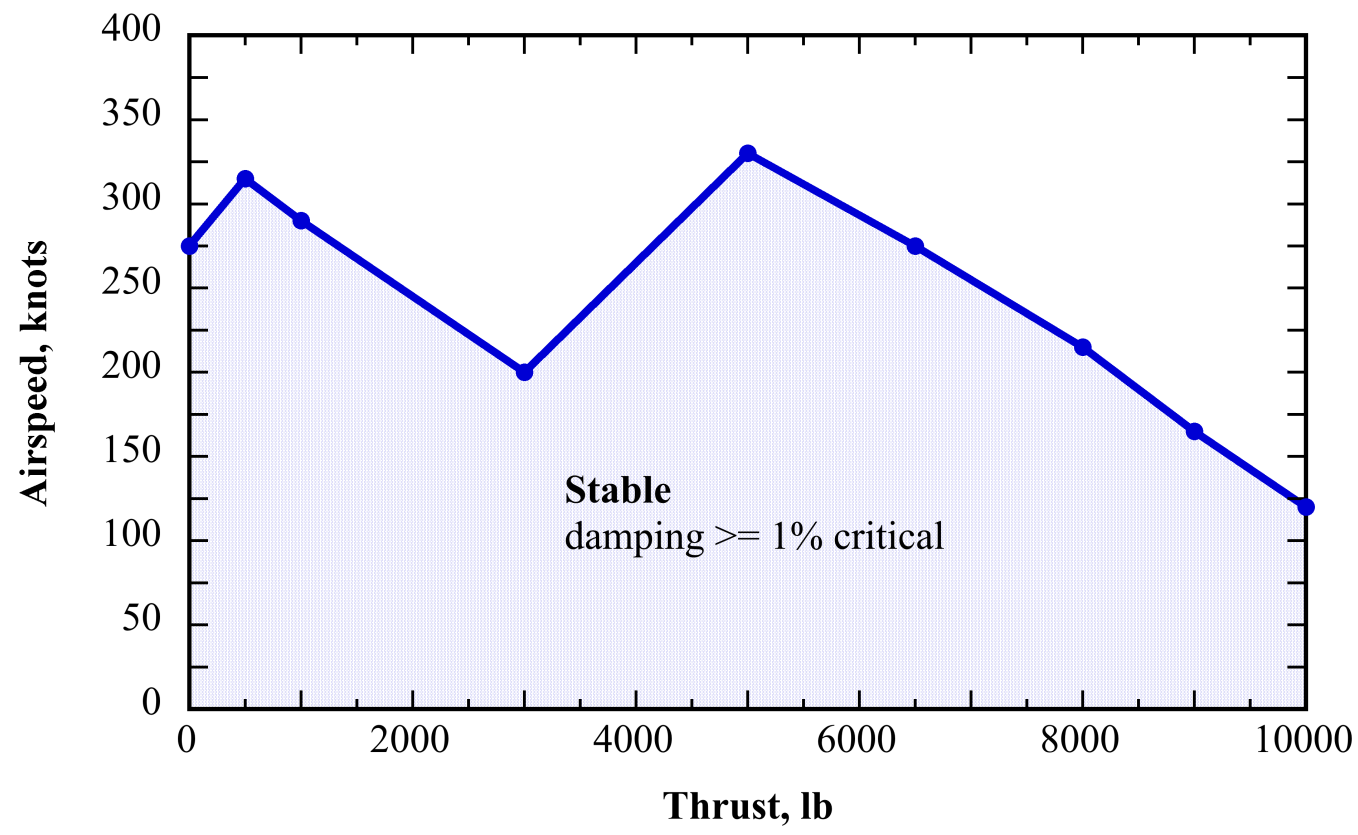

Fig. 11a. Predicted NASTRAN data-based TTR/699 helicopter mode 1\% damping stability envelope (90-deg yaw, 569 rpm).

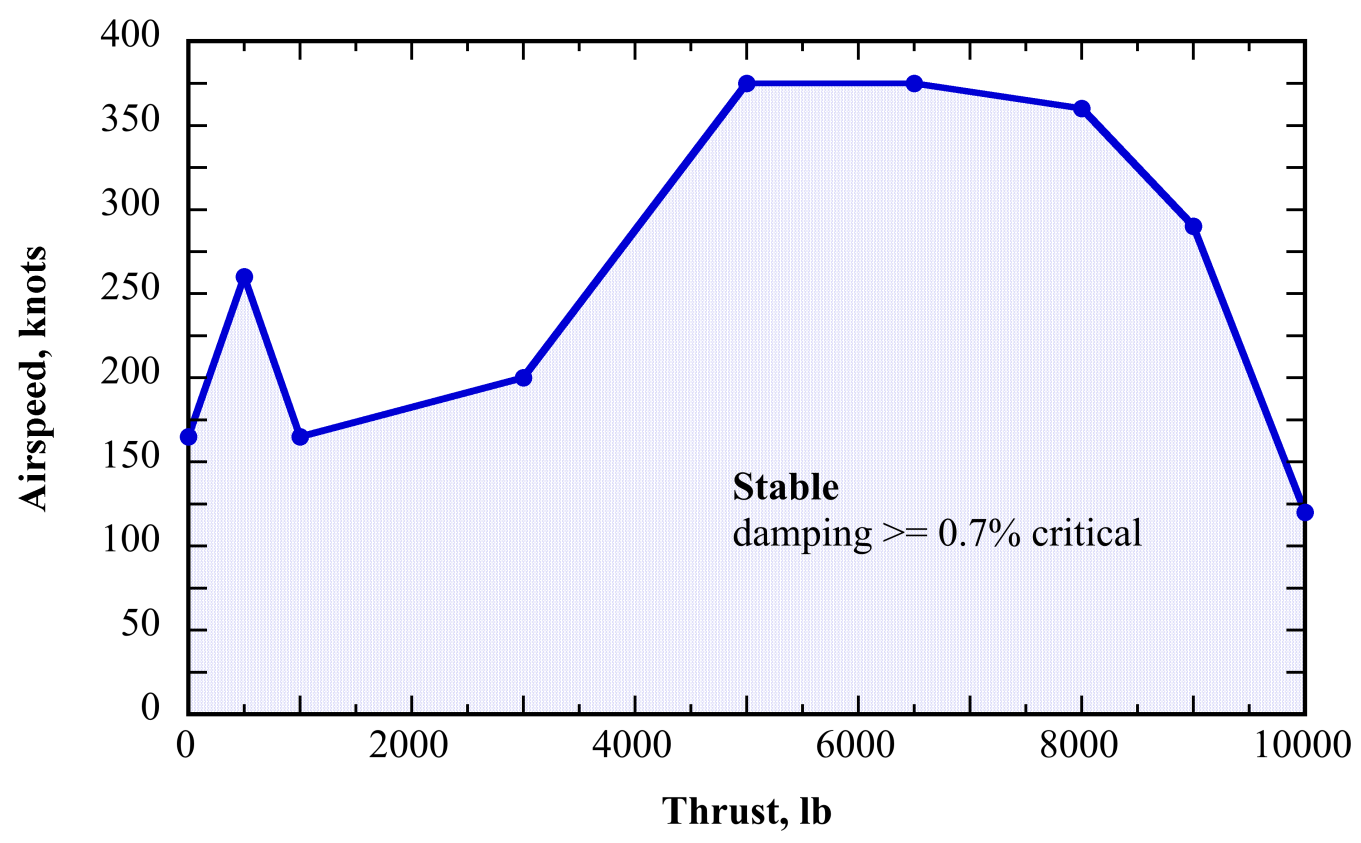

Fig. 11b. Predicted (worst-case) impact test data-based TTR/699 helicopter mode 0.7\% damping stability envelope (90-deg yaw, 569 rpm.) 


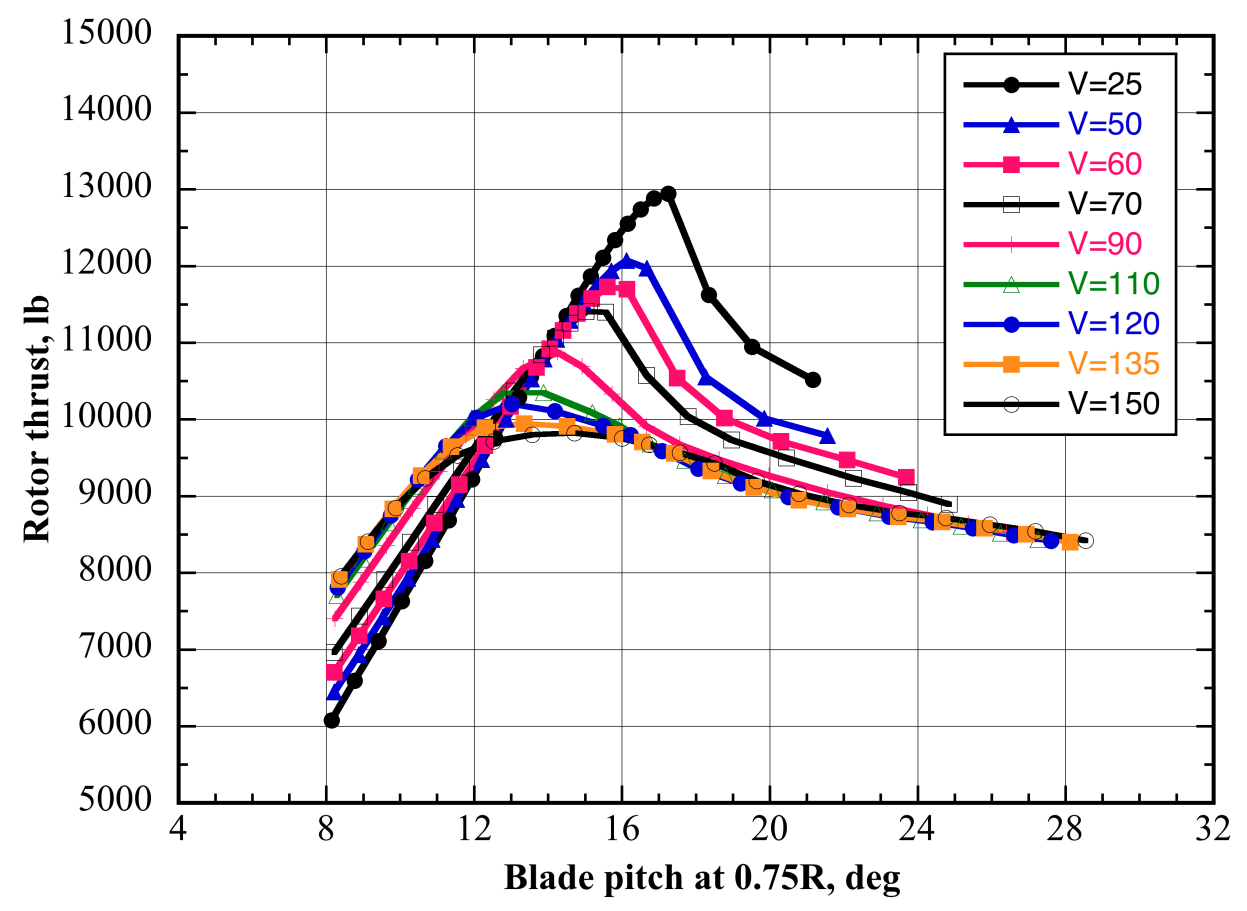

Fig. 12a. Predicted (high thrust) 699 helicopter mode thrust vs. collective (90-deg yaw, 569 rpm).

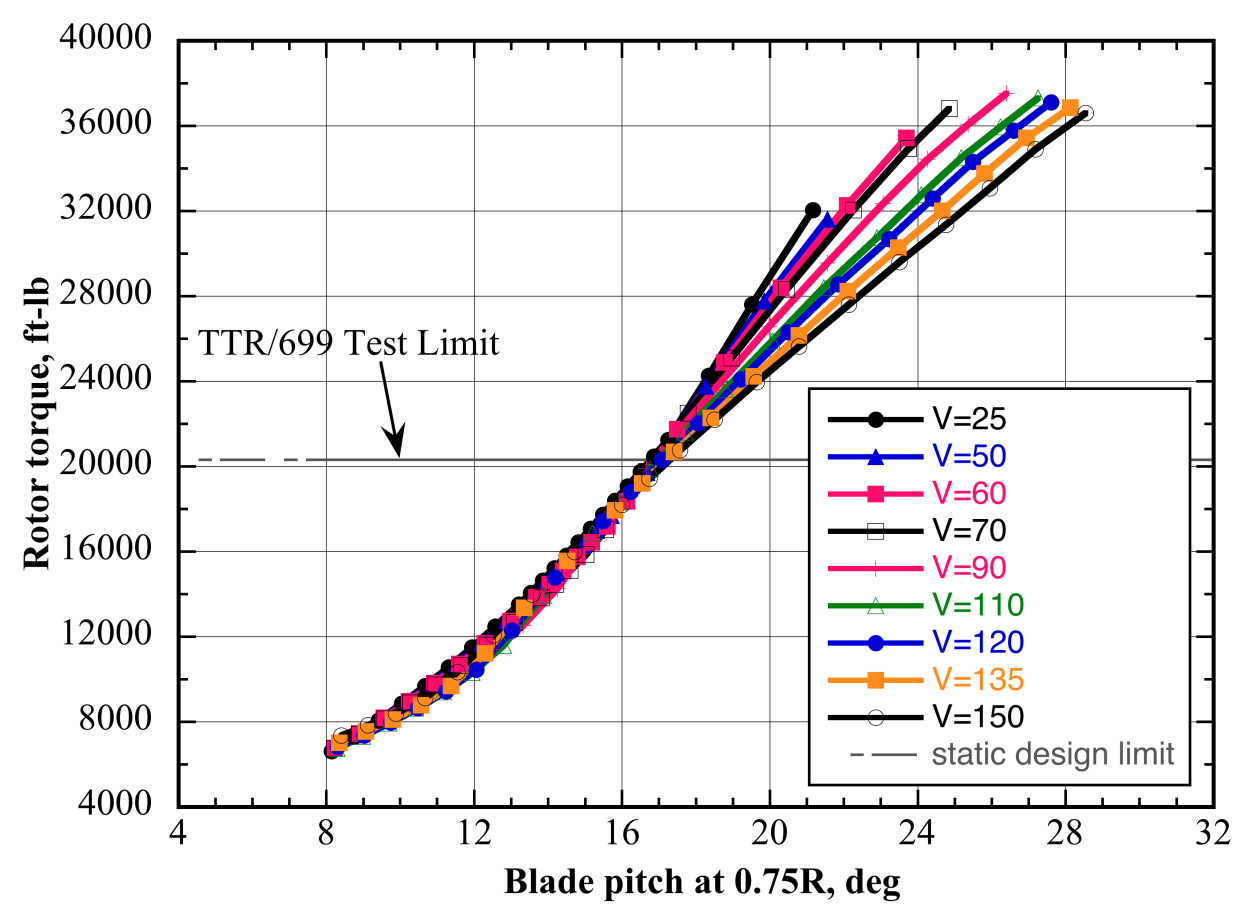

Fig. 12b. Predicted (high thrust) 699 helicopter mode torque vs. collective (90-deg yaw, 569 rpm). 


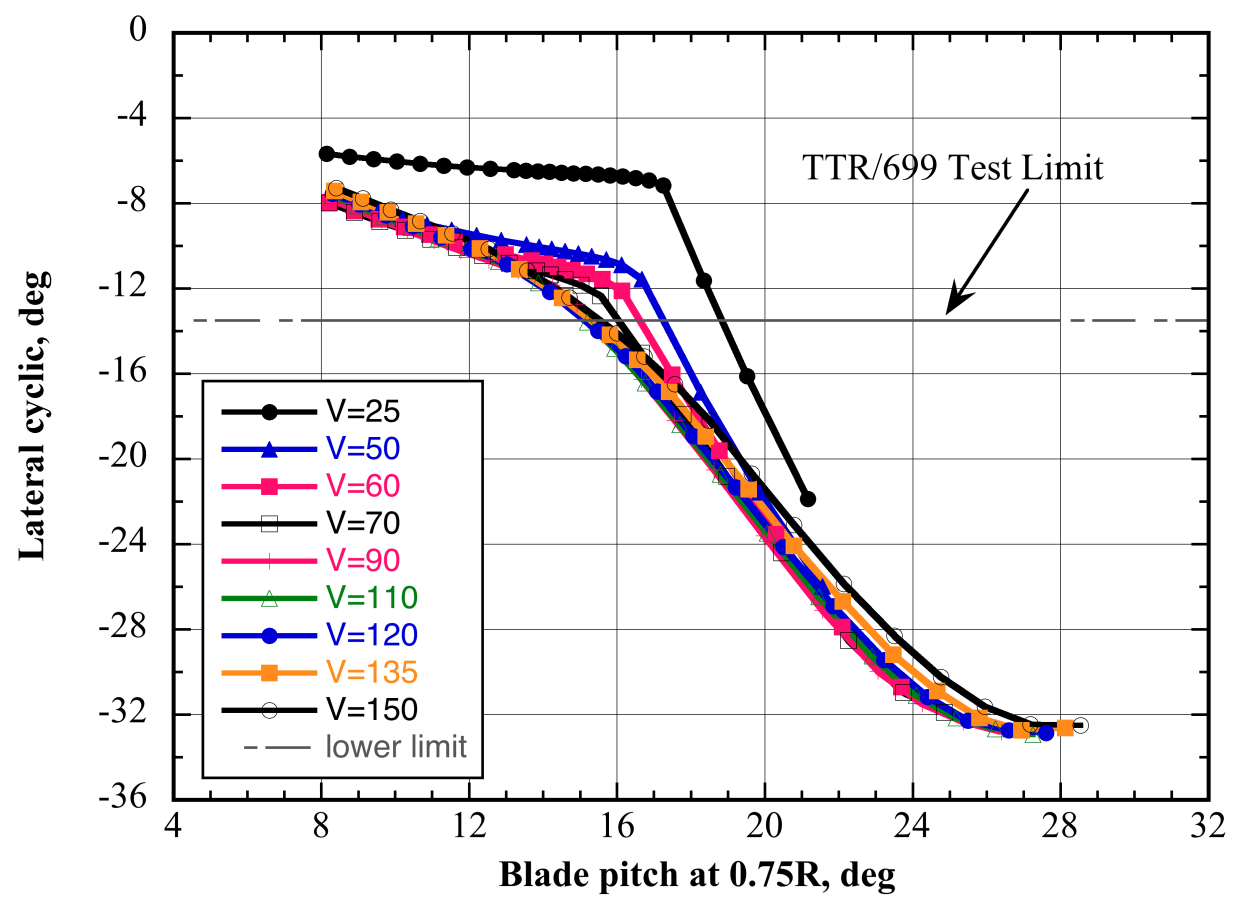

Fig. 12c. Predicted (high thrust) 699 helicopter mode lateral cyclic vs. collective (90-deg yaw, 569 rpm).

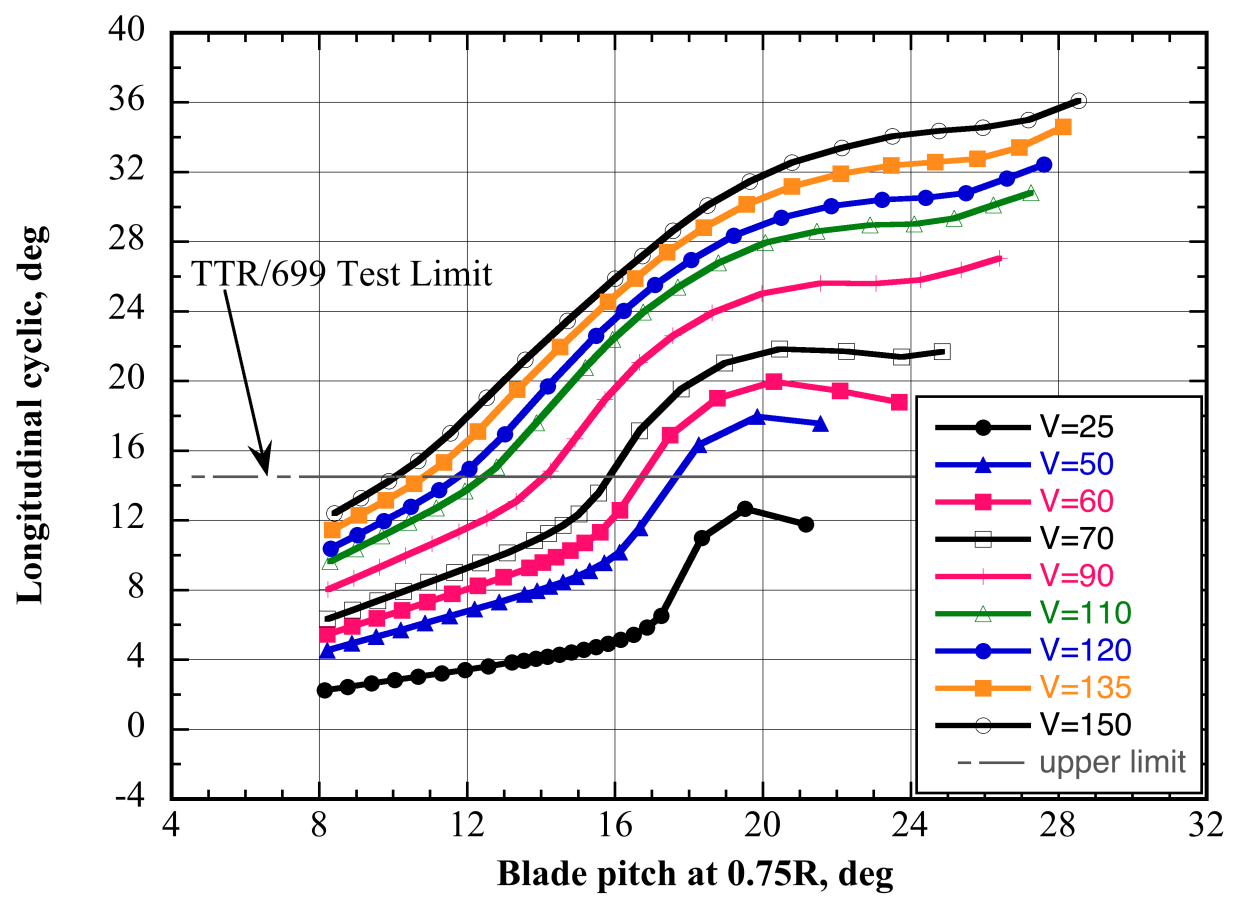

Fig. 12d. Predicted (high thrust) 699 helicopter mode longitunal cyclic vs. collective (90-deg yaw, 569 rpm). 


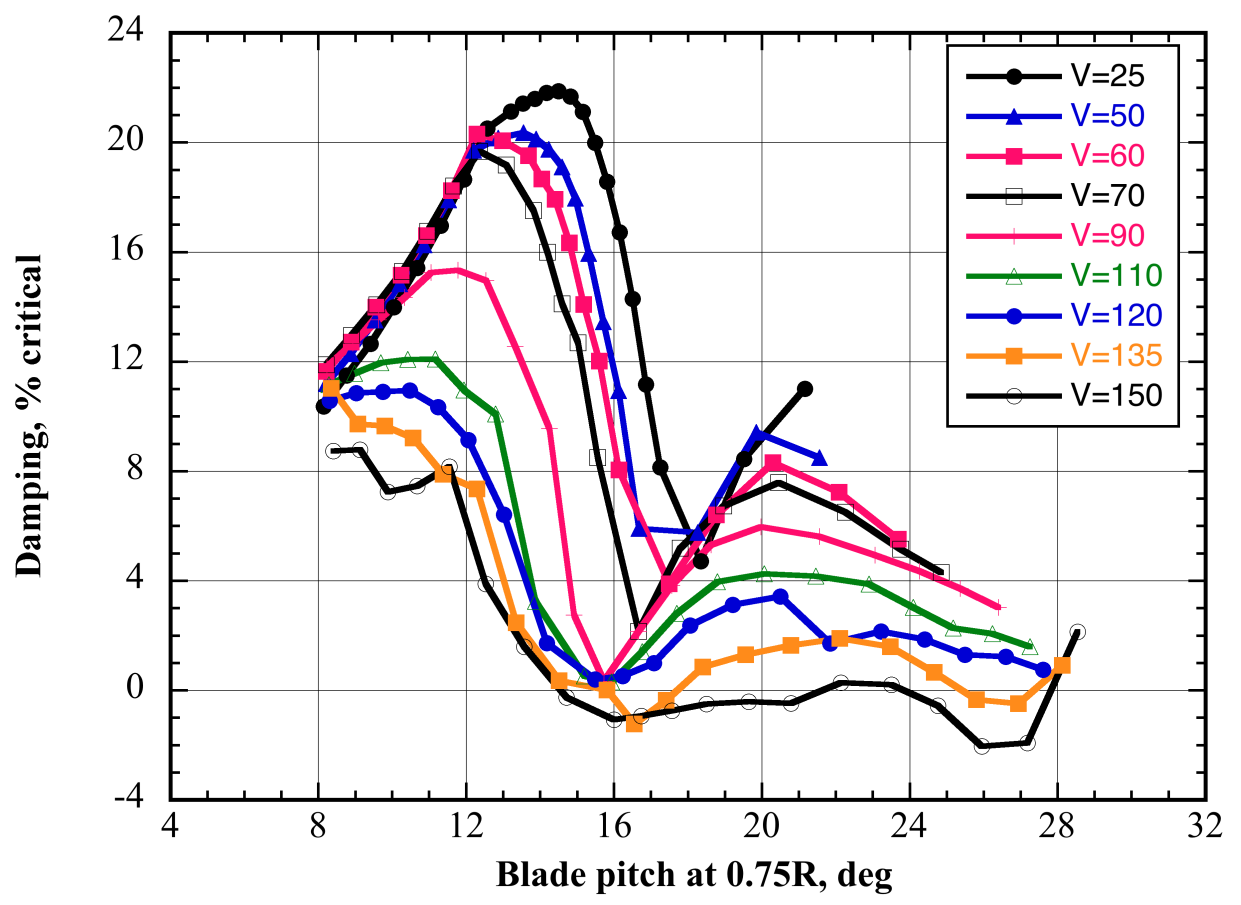

Fig. 13a. TTR/699 high thrust, worst-case nose yaw damping vs. collective, various airspeeds (90-deg yaw, $569 \mathrm{rpm})$.

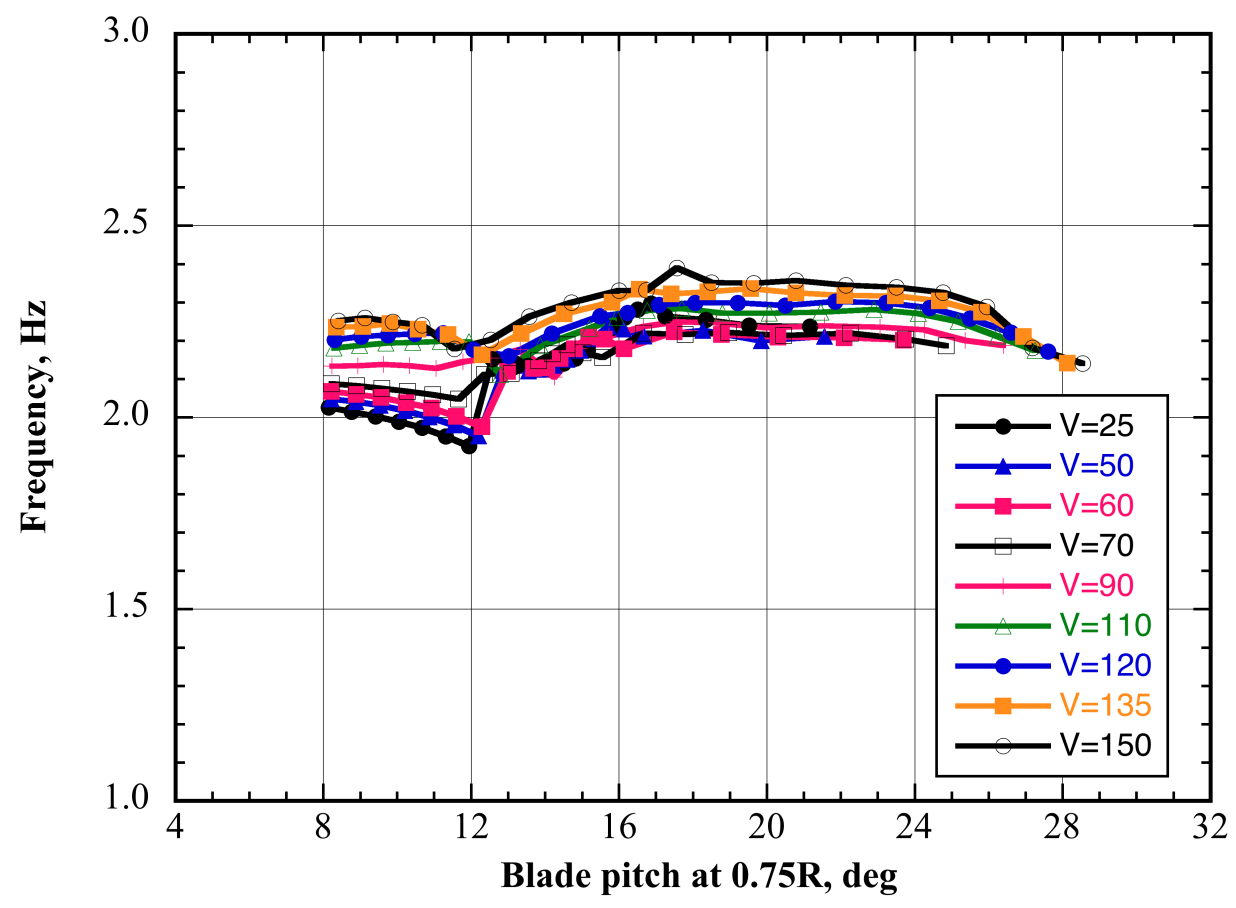

Fig. 13b. TTR/699 high thrust, worst-case nose yaw frequency vs. collective, various airspeeds (90-deg yaw, $569 \mathrm{rpm})$. 


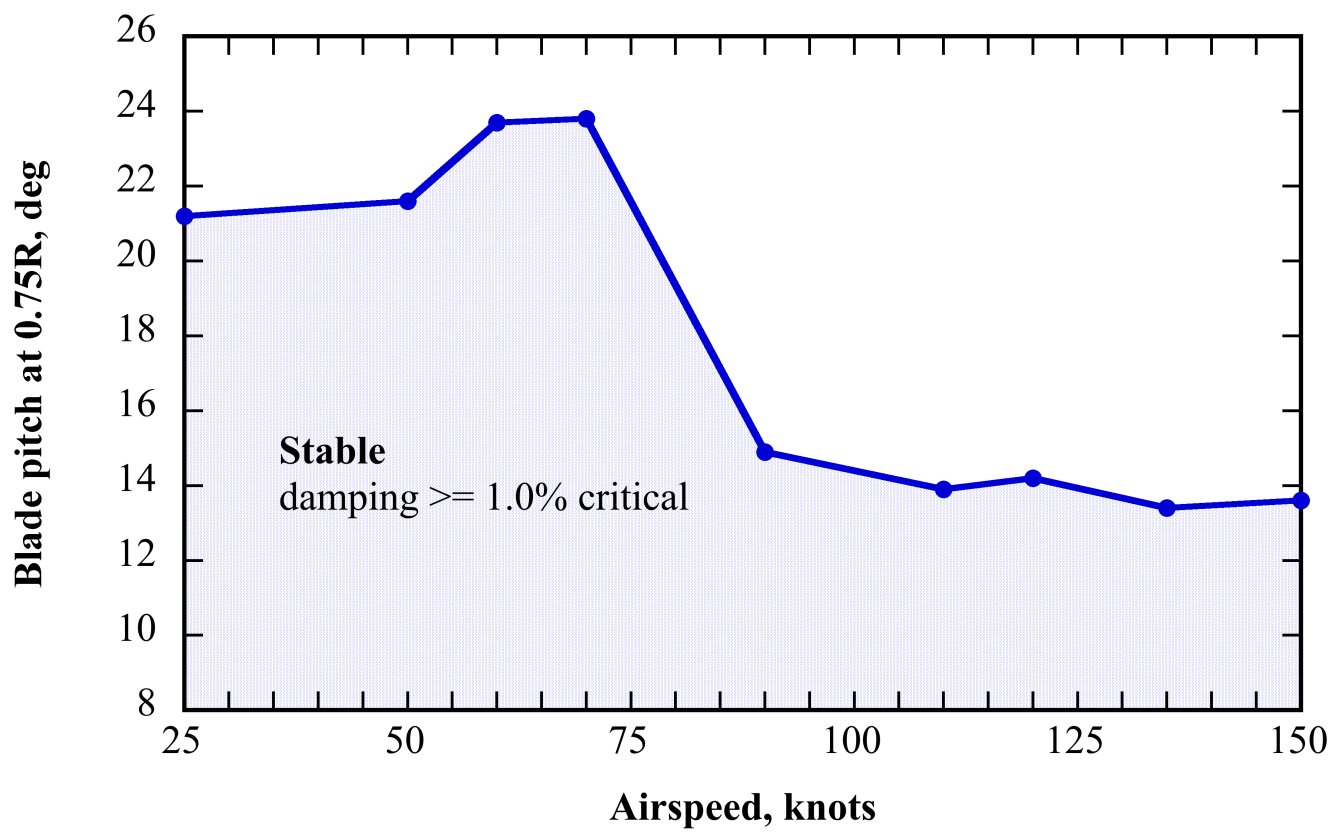

Fig. 14. Predicted high thrust, worst-case TTR/699 helicopter mode $1.0 \%$ damping stability envelope (90-deg yaw, 569 rpm).

The current stability predictions (hover, cruise, conversion, and helicopter mode) show that the TTR/699 is stable within the test envelope. It was found during the recently completed test that the calculated stability limits were well beyond the TTR test limits. During testing, for safety purposes the performance parameters (thrust, torque), blade loads, pitch link loads, and control system loads were monitored in real time. It turned out that the TTR test limits (especially for the torque and control system) were reached well before any stability limits.

\section{Conclusions}

Aeroelastic stability predictions for the Tiltrotor Test Rig (TTR) with the 699 research rotor installed in the USAF NFAC 40- by 80 -Foot Wind Tunnel were considered in this study. All test conditions were covered: hover, cruise (airplane mode), conversion, and helicopter mode. The TTR fixed-system dynamics were represented by modal data that were obtained from NASTRAN and from shake and impact tests of the TTR. Stability predictions were made using the comprehensive analysis CAMRAD II. Stability predictions were based on NASTRAN modal data and worstcase damping test data. The stability predictions showed that the TTR/699 is stable within the test envelope. The limiting mode is a strut mode specific to the NFAC installation; this limitation does not apply to the AW609 aircraft itself.

\section{Acknowledgments}

Bell Helicopter has been extensively involved with the Tiltrotor Test Rig right from its inception and also with the 699 research proprotor; the authors gratefully acknowledge the technical support given by Bell Helicopter.

\section{References}

[1] Russell, C. R. and Acree, C. W., "Modal Test and Analysis of the NASA Tiltrotor Test Rig," AHS Technical Conference on Aeromechanics Design for Transformative Vertical Lift, San Francisco, CA, January 16-19, 2018.

[2] Kottapalli, S. and Acree, C. W., "Analytical Performance, Loads, and Aeroelastic Stability of a Full-Scale Isolated Proprotor," AHS Technical Conference on Aeromechanics Design for Transformative Vertical Flight, San Francisco, CA, Jan 1619, 2018.

[3] Acree, C. W., Sheikman, A. L., Norman T. R., "High-Speed Wind Tunnel Tests of a Full-Scale Proprotor on the Tiltrotor Test Rig," submitted to the Vertical Flight Society VFS 75 ${ }^{\text {th }}$ Annual Forum, Philadelphia, PA, May 13-16, 2019. 
[4] Acree, C. W. and Sheikman, A. L., "Development and Initial Testing of the Tiltrotor Test Rig," AHS International 74th Annual Forum \& Technology Display, Phoenix, AZ, May 14-17, 2018.

[5] Kottapalli, S. and Acree, C. W., "Correlation of Full-Scale Isolated Proprotor Performance and Loads," submitted to the Vertical Flight Society VFS 75 ${ }^{\text {th }}$ Annual Forum, Philadelphia, PA, May 13-16, 2019.

[6] NASA Ames Aeromechanics website: https://rotorcraft.arc.nasa.gov/Research/Facilities/ttr.html .

[7] Johnson, W., "CAMRAD II, Comprehensive Analytical Model of Rotorcraft Aerodynamics and Dynamics," Johnson Aeronautics, Palo Alto, California, 1992-1999.

[8] Johnson, W., "Technology Drivers in the Development of CAMRAD II," American Helicopter Society, American Helicopter Society Aeromechanics Specialists Conference, San Francisco, CA, January 19-21, 1994.

[9] Johnson, W., “A General Free Wake Geometry Calculation for Wings and Rotors,” American Helicopter Society 51st Annual Forum Proceedings, Ft. Worth, TX, May 9-11, 1995.

[10] Solis, E., and Meyn, L., "Photogrammetric Deflection Measurements for the Tiltrotor Test Rig (TTR) Multi-Component Rotor Balance Calibration," American Helicopter Society Technical Meeting on Aeromechanics Design for Vertical Lift, San Francisco, CA, January 20-22, 2016

[11] Acree, C. W. Jr., “A CAMRAD II Model of the V-22 Rotor for Whirl-Flutter Analysis”, NASA TM 2004-212801, July 2004.

[12] Harris, J.C., Scheidler, P.F, Hopkins, R, and Fortenbaugh, R.L., "Initial Power-Off Testing of the BA609 Tiltrotor," American Helicopter Society 66th Annual Forum Proceedings, Phoenix, AZ, May 11-13, 2010.

[13] Parham, T. and Corso, L. M., “Aeroelastic and Aeroservoelastic Stability of the BA 609," 25th European Rotorcraft Forum, September 1999. 